\title{
Effect Analysis of Early Warning for Abandoned Object on Highway Based on Internet-of-Vehicles CA Model
}

\author{
Juan Bao, ${ }^{1}$ Wei Chen $\mathbb{D}^{1},{ }^{1}$ Zhengtao Xiang $\mathbb{D}^{2},{ }^{2}$ Yufeng Chen $\mathbb{D},{ }^{2}$ and Yishui Shui ${ }^{3}$ \\ ${ }^{1}$ School of Automation, Wuhan University of Technology, Wuhan 430070, China \\ ${ }^{2}$ School of Electrical and Information Engineering, Hubei University of Automotive Technology, Shiyan 442002, China \\ ${ }^{3}$ School of Information Engineering, Wuhan University of Technology, Wuhan 430070, China \\ Correspondence should be addressed to Wei Chen; greatchen@whut.edu.cn
}

Received 11 October 2017; Accepted 21 December 2017; Published 22 January 2018

Academic Editor: Tetsuji Tokihiro

Copyright (C) 2018 Juan Bao et al. This is an open access article distributed under the Creative Commons Attribution License, which permits unrestricted use, distribution, and reproduction in any medium, provided the original work is properly cited.

\begin{abstract}
An early warning on the highway will effectively reduce traffic accidents. Considering the influence of an abandoned object on driving behavior, a Visual-based Asymmetric Two-lane Cellular Automata model with Abandoned Object (V-ATCA-AO) and an Internet-of-Vehicles-based Asymmetric Two-lane Cellular Automata model with Abandoned Object (IoV-ATCA-AO) are proposed. Based on the two models, two types of traffic accidents caused by an abandoned object are analyzed: rear-end collision caused by the abandoned object ahead and collision of the vehicle with the abandoned object. Simulation results show the following: (1) the accidents occur when the road density is smaller, while the accidents will not occur when the density is larger. The results are different from the rear-end collision rate curve without abandoned object in a single lane. (2) Compared with the visual-based avoidance pattern in V-ATCA-AO, the Internet-of-Vehicles-based avoidance pattern in IoV-ATCA-AO can create an early warning for the abandoned object and tell the vehicle to make an earlier lane change and decelerate in advance, thereby significantly reducing the accident rate. (3) Spatiotemporal characteristics in front of the abandoned object directly affect the accident rate: the less the "stability" of a traffic jam in front of the abandoned object, the higher the accident rate.
\end{abstract}

\section{Introduction}

The study of abandoned objects on the highway mainly includes abandoned objects from humans and abandoned objects from vehicles. Abandoned objects from humans refer to the various discarded waste from drivers and passengers such as garbage bags and bottles. Abandoned objects from vehicles refer to fallen vehicle parts or goods from vehicles, which result from a lack of maintenance or overloaded trucks; these objects include such things as tires and stones. On the highway, the sudden appearance of an abandoned object can seriously interfere with driving behavior and cause a traffic accident such as a rear-end collision. If the driver receives an early warning of the abandoned object, they will have time to adjust their driving behavior, including deceleration and lane changing in advance and thereby reducing the occurrence of traffic accidents.

To create the early warning for the abandoned object, the following two items need to be addressed: (1) Abandoned
Object Detection. Abandoned object detection falls under traffic accident detection, which is divided into the two major categories of artificial detection and automatic detection [1]. (2) Information Release regarding the Abandoned Object. Before the abandoned object can be cleaned, the traffic control center uses various technical means to push the information about the abandoned object to vehicles approaching the object on the highway. These vehicles are called "affected vehicles."

Issuing effective early warnings in real time by traditional early warning methods is difficult; traffic accidents are often caused by the abandoned object. (1) Abandoned Object Detection. Artificial detection methods mainly include object detection by a driver calling the police, the traffic patrol car on patrol, surveillance video, or artificial recognition by a traffic control center. These methods are inadequate because of poor timing and inaccurate descriptions. Automatic detection is mainly based on image processing techniques. The 
advantages of automatic recognition include fast detection and high accuracy; however, to achieve full coverage of a highway, a lot of cameras must be installed at high cost. In practice, cameras are usually installed only in subsections of the highway. (2) Information Release about Abandoned Object. Traditional alert methods include using a changeable message sign or traffic radio, for example. The general problem with these methods includes the fact that the rate information is received by the affected vehicles. The scope of the information board method is limited by the number and location of the installation. At the same time, at high speeds, drivers may not pay much attention to the details of the changeable message signs. Similarly, the effectiveness of traffic radio is limited to the drivers listening to relevant channels.

In recent years, the development of Internet-of-Vehicles technology offers a new solution for early warnings of abandoned objects. Internet-of-Vehicles technology can greatly improve the traffic safety [2]. Using the Internet-of-Vehiclesbased warning pattern for an abandoned object, vehicles in the road communicate with the traffic control center using the vehicle terminal (or mobile app) to upload and receive information. (1) Abandoned object detection. The lane changing behavior of the vehicle is detected by the gyroscope in the vehicle terminal, and the behavior information is uploaded to the traffic control center. At the same time, GPS position and velocity are also uploaded in real time while the vehicle travels. Using the information uploaded by the vehicle, the traffic control center determines whether an abandoned object is on the road. The assessment is made by the following: if the lane changing behavior occurs when passing a certain location and no vehicle is present in that location, it can be determined that an abandoned object is at the location. (2) Information Release about Abandoned Object. Based on the GPS location uploaded by the vehicles, the spatial distribution of vehicles on the road is calculated by a traffic control center. Thus, position information is pushed to the affected vehicles and the warning is used by the affected vehicle terminals to prompt drivers to slow down and change lane in advance.

Therefore, compared with traditional early warning methods for abandoned objects, the Internet-of-Vehiclesbased warning method for abandoned object seems better: in terms of detection of abandoned objects, this method not only occurs quickly and with high accuracy, but also can guarantee the integration of the detection. In terms of information release, the method can guarantee that affected vehicles with a vehicle terminal will be able to receive warning information about the abandoned object.

To study the reducing effects of a warning threshold for an abandoned object on the accident rate based on the Internet of Vehicles, in this article we first consider the influence of the abandoned object on driving behavior and propose both a Visual-based Asymmetric Two-lane Cellular Automata model with Abandoned Object (V-ATCA-AO) and an Internet-of-Vehicles-based Asymmetric Two-lane Cellular Automata model with Abandoned Object (IoV-ATCA-AO). Two types of traffic accidents caused by the abandoned object are analyzed and the corresponding criteria for accidents are proposed. Finally, with spatiotemporal characteristics of traffic flow, accident rate characteristics of two types of models are simulated and the effect of the warning threshold for abandoned objects on the accident rate based on Internet-ofVehicles is also analyzed. The research provides a reference for the creation of an immediate warning system for abandoned object.

\section{Models}

2.1. Basic Two-Lane CA Model. The dynamic evolution process of traffic flow can be obtained by the CA model [3-12]. Time, space, and vehicle state (such as velocity) are discrete in the CA model. In the progress of time step $t \rightarrow t+1$, all vehicles are dynamically updated on the road according to local rules. In a typical single-lane Nagel-Schreckenberg (NS) model [3], the road is divided into cells and the length is $L$, and every cell is occupied by one vehicle or empty. $x(k, t)$ and $v(k, t)$ denote the position and velocity, respectively, of vehicle $k$ at the time step $t . v_{\max }$ denotes the maximum velocity. $d(k, t)=x(k+1, t)-x(k, t)-1$ denotes the gap between vehicle $k$ and the preceding vehicle $k+1$. The rules are listed below.

(1) Acceleration is

$$
v(k, t+1)=\min \left(v(k, t)+1, v_{\max }\right) .
$$

(2) Slowing down is

$$
v(k, t+1)=\min (v(k, t+1), d(k, t)) .
$$

(3) Randomization is

$$
\begin{gathered}
\text { with probability } p, v(k, t+1) \\
=\max (v(k, t+1)-1,0) .
\end{gathered}
$$

(4) Vehicle motion is

$$
x(k, t+1)=x(k, t)+v(k, t+1) .
$$

Lane changing rules are added on the basis of the NS model, and a two-lane CA model [13-19] is established. Considering different lanes and vehicles, lane changing rules are divided into two categories: symmetrical lane changing rules and asymmetric lane changing rules. In symmetric twolane cellular automata (STCA) proposed by Chowdhury et al., lane changing rules consist of two parts: incentive for the lane change and safety criterion [13]. The incentive for the lane change consists of two parts: (1) the vehicle can not travel at the expected speed in this lane; (2) the travel condition of the neighboring lane is better than that of this lane. The safety criterion is to ensure that the lane changing process does not cause a crash. Lane changing rules in the STCA model are as follows.

(1) Incentive for the lane change is

$$
\begin{aligned}
d(k, t) & <\min \left(v(k, t)+1, v_{\max }\right), \\
d_{\text {pred }}(k, t) & >d(k, t) .
\end{aligned}
$$




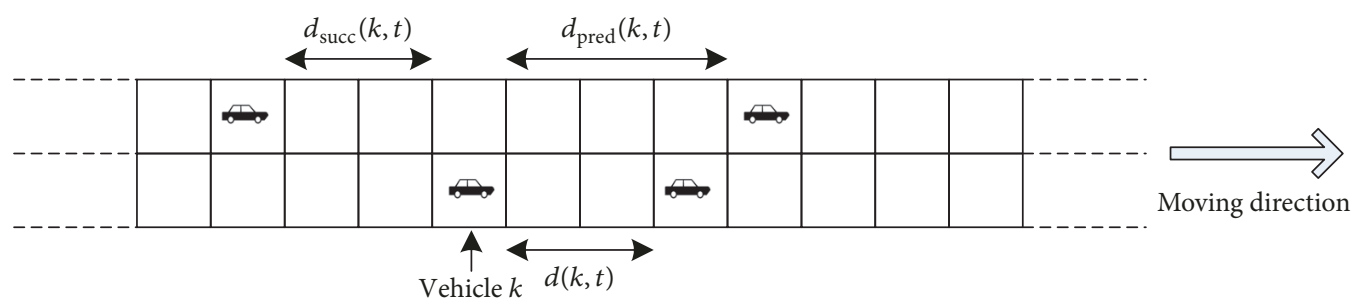

FIGURE 1: Corresponding positions of vehicles for lane changing rules in the STCA model.

(2) Safety criterion is

$$
d_{\text {succ }}(k, t)>d_{\text {safe }} .
$$

Corresponding positions of the vehicles in the STCA model are shown in Figure 1 . Here, $d_{\text {pred }}(k, t)$ denotes the gap between vehicle $k$ and the preceding vehicle in the neighboring lane. $d_{\text {succ }}(k, t)$ denotes the gap between vehicle $k$ and the succeeding vehicle in the neighboring lane. $d_{\text {safe }}$ is the security gap.

In the two-lane CA model, the rules are updated in two steps: first step, vehicles complete lane changes according to lane changing rules; second step, vehicles complete velocity update and movement according to the NS model.

2.2. Visual-Based Two-Lane Cellular Automata Model with Abandoned Object. Abandoned objects can appear in any lane; the model in this article assumes that the abandoned object is in the right lane. Vehicles can directly pass through small abandoned objects with minimal effect on the traffic; for large abandoned objects, vehicles must change lanes to bypass the object, resulting in a greater influence on the traffic.

This study assumes that the abandoned object is a large one. Vehicles must change lanes to bypass the abandoned object and the left and right lanes have different lane changing rules. Therefore, asymmetric lane changing rules are used in the two-lane model with abandoned object. If there is no vehicle terminal in the vehicle, the abandoned object can only be found through the vision of the driver. The two-lane CA model with abandoned object in this kind of scenario is called a "Visual-based Asymmetric Two-lane Cellular Automata model with Abandoned Object" (V-ATCA-AO). If there is a vehicle terminal in the vehicle, the abandoned object can be discovered in advance through the vehicle terminal. The two-lane CA model with abandoned object in this kind of scenario is called an "Internet-of-Vehicles-based Asymmetric Two-lane Cellular Automata model with Abandoned Object" (IoV-ATCA-AO).

In V-ATCA-AO, the driver does not know in advance about the abandoned object ahead. When the driver can not see the abandoned object, the driving behavior is the same as the behavior with the STCA model. When the abandoned object appears in view, driving behavior differs from that in the STCA model. Based on the STCA model, lane changing rules of V-ATCA-AO are proposed.
Changing rules in the left lane are as follows.

(1) Incentive for the lane change is

$$
\begin{aligned}
& \operatorname{not}\left(x(k, t)<x_{\mathrm{ao}}<x_{\text {pred }}(k, t), d_{\mathrm{ao}}(k, t)<d_{\mathrm{v}}\right), \\
& \left(d(k, t)<\min \left(v(k, t)+1, v_{\mathrm{max}}\right), d_{\text {pred }}(k, t)\right. \\
& \quad>d(k, t)) .
\end{aligned}
$$

(2) Safety criteria are

$$
\begin{gathered}
\left(x(k, t)>x_{\mathrm{ao}}, x_{\text {succ }}(k, t)<x_{\mathrm{ao}}\right) \\
\text { or }\left(d_{\text {succ }}(k, t)>d_{\text {safe }}\right) .
\end{gathered}
$$

The positions of the vehicles and the abandoned object for left lane changing rules in V-ATCA-AO are shown in Figure 2. $x_{\mathrm{ao}}$ denotes the location of the abandoned object, and $d_{\mathrm{ao}}(k, t)$ denotes the gap between vehicle $k$ and the abandoned object ahead; $x_{\text {pred }}(k, t)$ and $x_{\text {succ }}(k, t)$ denote the location of the preceding vehicle and the successor vehicle, respectively, in the neighboring lane at the time step $t$. In addition, in the above formulas, $d_{\mathrm{v}}$ means the visible distance of the abandoned object, that is to say, the distance to the abandoned object seen by the driver.

As for the incentive for the lane change, when the driver in the left lane sees the abandoned object ahead in the right lane, he will cancel the incentive for changing a lane; the conditions $x(k, t)<x_{\mathrm{ao}}<x_{\text {pred }}(k, t)$ and $d_{\mathrm{ao}}(k, t)<d_{\mathrm{v}}$ for the incentive describe this situation. When he is not able to see the abandoned object ahead in the right lane, the incentive for the lane change is the same as that in the STCA model.

For the safety criteria, in addition to safety criteria of the STCA model, another case has been described by $x(k, t)>$ $x_{\mathrm{ao}}$ and $x_{\text {succ }}(k, t)<x_{\mathrm{ao}}$ : the vehicle in the left lane has passed over the abandoned object, but the succeeding vehicle in the right lane is blocked by the abandoned object.

Lane changing rules in right lane are as follows.

(1) Incentive for the lane change is

$$
\begin{aligned}
& \left(x(k, t)<x_{\mathrm{ao}}<x(k+1, t), d_{\mathrm{ao}}(k, t)<d_{\mathrm{v}}\right) \text { or } \\
& \left(d(k, t)<\min \left(v(k, t)+1, v_{\max }\right), d_{\text {pred }}(k, t)\right. \\
& \quad>d(k, t)) .
\end{aligned}
$$




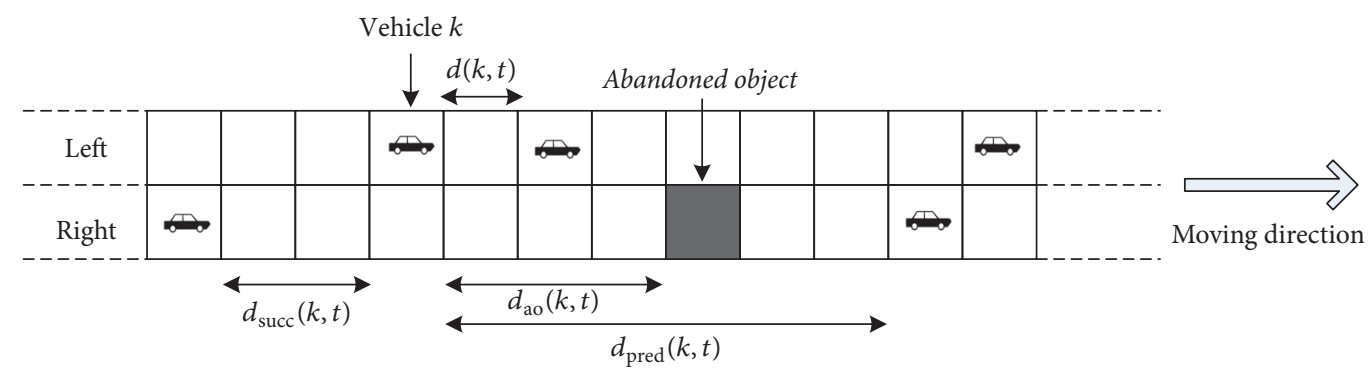

Figure 2: Positions of vehicles and the abandoned object for left lane changing rules in V-ATCA-AO.

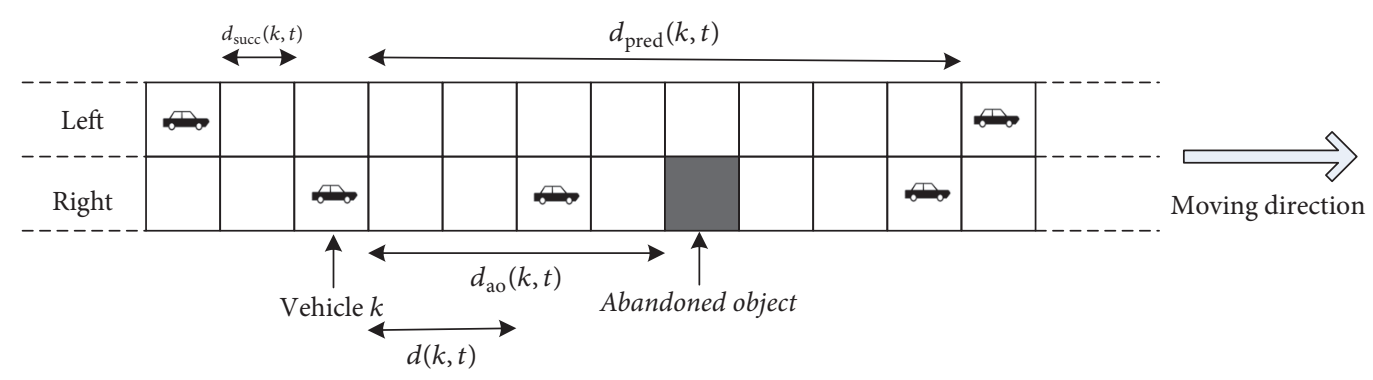

FIgURE 3: Positions of vehicles and the abandoned object for right lane changing rules in V-ATCA-AO.

(2) Safety criterion is

$$
d_{\text {succ }}(k, t)>d_{\text {safe }}
$$

In Figure 3, the positions of the vehicles and the abandoned object are given, with lane changing rules in the right lane in V-ATCA-AO. Regarding the incentive for the lane change, the sight of the abandoned object ahead in the right lane will generate the incentive for changing a lane, and conditions $x(k, t)<x_{\mathrm{ao}}<x(k+1, t)$ and $d_{\mathrm{ao}}(k, t)<$ $d_{\mathrm{v}}$ describe this situation. When the driver cannot see the abandoned object ahead in the lane, the incentive for the lane change remains the same as in the STCA model. The safety criterion for lane changing rules in the right lane is also the same as in the STCA model.

According to the lane changing rules above, when the driver in the right lane sees the abandoned object ahead, if safety conditions are not satisfied, the driver will not be able to change a lane. In this case, if the velocity of this vehicle is high, the driver will quickly slow down to avoid hitting the abandoned object. This phenomenon can be realized by modifying the maximum velocity $v_{\max }$ of the NS model. Its means that, for vehicles in the right lane when the conditions $d_{\mathrm{ao}}(k, t)<d_{\mathrm{v}}$ and $x(k+1, t)>x_{\mathrm{ao}}$ are satisfied, the maximum velocity is $v_{\max }-2$.

\subsection{Internet-of-Vehicles-Based Two-Lane Cellular Automata} Model with Abandoned Object. Based on Internet-of-Vehicles technology, when the gap between the vehicle and the abandoned object is less than a certain threshold, the vehicle terminal sends out a warning according to the length of the gap between the abandoned object and the vehicle, to remind the driver to decelerate and change lanes in advance. At this time, the incentive for the lane change in the left lane is not satisfied, while the incentive for the lane change in the right lane is satisfied. The warning principles are shown as follows.

(1) When $d_{\mathrm{v} 2 \mathrm{v}}^{2}<d_{\mathrm{ao}}(k, t) \leq d_{\mathrm{v} 2 \mathrm{v}}^{1}$, a vehicle travels in the normal warning zone. In this warning zone, the vehicle terminal reports to the driver that there is an abandoned object ahead in the right lane and the gap from the vehicle is $d_{\mathrm{ao}}(k, t)$; the vehicle terminal reminds the driver to travel on the left, and the maximum velocity in the right lane is set to $v_{\max }-1$ while the maximum velocity in the left lane remains unchanged.

(2) When $d_{\mathrm{ao}}(k, t) \leq d_{\mathrm{v} 2 \mathrm{v}}^{2}$, a vehicle travels in the emergency warning zone. In this warning zone, the vehicle terminal reports to the driver that there is an abandoned object ahead in the right lane and the gap from the vehicle is $d_{\mathrm{ao}}(k, t)$; the vehicle terminal reminds the driver to travel on the left, and the maximum velocity in the right lane is set to $v_{\max }-2$ while the maximum velocity in the left lane remains unchanged.

In this case, $d_{\mathrm{v} 2 \mathrm{v}}^{1}$ and $d_{\mathrm{v} 2 \mathrm{v}}^{2}$ denote two warning thresholds for different stage in the vehicle terminal.

Based on the above warning principle, lane changing rules of IoV-ATCA-AO are proposed on the basis of the STCA model.

Lane changing rules in the left lane are as follows:

(1) Incentive for the lane change is

$$
\begin{aligned}
& \operatorname{not}\left(x(k, t)<x_{\mathrm{ao}}, d_{\mathrm{ao}}(k, t)<d_{\mathrm{IoV}}^{1}\right), \\
& \left(d(k, t)<\min \left(v(k, t)+1, v_{\max }\right), d_{\mathrm{pred}}(k, t)\right. \\
& \quad>d(k, t)) .
\end{aligned}
$$


(2) Safety criteria are

$$
\begin{gathered}
\left(x(k, t)>x_{\mathrm{ao}}, x_{\text {succ }}(k, t)<x_{\mathrm{ao}}\right) \\
\text { or }\left(d_{\text {succ }}(k, t)>d_{\text {safe }}\right) .
\end{gathered}
$$

Lane changing rules in the right lane are as follows:

(1) Incentive for the lane change is

$$
\begin{aligned}
& \left(x(k, t)<x_{\mathrm{ao}}, d_{\mathrm{ao}}(k, t)<d_{\mathrm{IoV}}^{1}\right) \text { or } \\
& \left(d(k, t)<\min \left(v(k, t)+1, v_{\max }\right), d_{\mathrm{prec}}(k, t)\right. \\
& \quad>d(k, t)) .
\end{aligned}
$$

(2) Safety criterion is

$$
d_{\text {succ }}(k, t)>d_{\text {safe }}
$$

In IoV-ATCA-AO, when the vehicle has not entered into the warning zone, the vehicle terminal will not send out a warning. At this time, the conditions that satisfy the incentive for a lane change are the same with the STCA model. The conditions $\left(x(k, t)<x_{\mathrm{ao}}\right.$ and $\left.d_{\mathrm{ao}}(k, t)<d_{\mathrm{IoV}}^{1}\right)$ of the incentive for the lane change describe the situation in which the vehicle enters into the warning zone. Safety criteria for the lane change are the same as with V-ATCA-AO model.

\section{Judgment Conditions of Traffic Accident}

In the NS model, rule (2) ensures the accident will not occur; that is, all drivers will observe the safety gap. However, in actual traffic, not all drivers will drive carefully, so traffic accidents occur regularly. Some researchers have proposed a dangerous situation (DS) scenario [20-23] within the framework of the NS model, which can be used to judge the occurrence of traffic accidents.

Within the framework of the deterministic NS model (randomization with probability $p=0$ ), Boccara et al. studied rear-end collisions caused by careless drivers with an ignorance of the safety gap [20]. The probability of the careless driver is assumed to be $p_{\text {col }}$, and the value of the probability is assumed to be irrelevant to the time and vehicle. At the time step $t$, when the predecessor is in a motion state $v(k+1, t)>0$, the careless driver of the successor will be expecting that their predecessor will continue to move forward at the next step $t+1$; that is, $v(k+1, t+1)>0$. Therefore, the careless driver will add 1 to the velocity on the basis of rule (2). In this case, if the predecessor suddenly stops at the next step $t+1$, a rear-end collision may be caused by the successor, the careless driver. Based on this assumption, Boccara et al. proposed three conditions of the DS [20]:

$$
\begin{aligned}
& \text { (i) } d(k, t) \leq v_{\max } \\
& \text { (ii) } v(k+1, t)>0 \\
& \text { (iii) } v(k+1, t+1)=0 .
\end{aligned}
$$

Notice that, in the process of simulation, the rules of the vehicle have been updated by using the NS model, so the traffic accident will not really occur. The above judgment conditions of DS are only used to determine whether the traffic accident occurs and cannot change the rules of the NS model.

In a subsequent study, Yang and Ma modified conditions (i) first proposed by Boccara and proposed conditions of the DS [21] within the framework of the nondeterministic NS model (randomization with probability $p \neq 0$ ):

$$
\begin{aligned}
& \text { (i) } v(k, t+1)=d(k, t) \\
& \text { (ii) } v(k+1, t)>0 \\
& \text { (iii) } v(k+1, t+1)=0 \text {. }
\end{aligned}
$$

The judgment conditions for DS proposed by Boccara et al. and Yang assume that the predecessor suddenly stops at the next time step. In fact, in addition to a sudden stop of the predecessor, sudden and great deceleration is also a significant cause of traffic accidents. Moussa introduced the concept of reaction time $\tau$ and proposed the judgment conditions for DS in this case [23].

$$
\begin{aligned}
& \text { (i) } \tau v(k, t)>d(k, t)+v(k+1, t+1) \\
& \text { (ii) } v(k+1, t)-v(k+1, t+1) \geq v_{d}
\end{aligned}
$$

The reaction time $\tau$ is defined as follows: when the predecessor slows down with the brake light on, the successor will reduce velocity to avoid a rear-end collision. The reaction time is the difference between the instant the brake light of the predecessor goes on and when the successor begins his braking maneuver [23]. Condition (i) in (17) denotes that, in the reaction time $\tau$, vehicle $k$, if it does not slow down, will drive to the location of vehicle $k+1$. That is to say, the succeeding vehicle will crash into the preceding vehicle. Condition (ii) denotes that vehicle $k+1$ at the next time step $t+1$ greatly and suddenly slows. This phenomenon of a sudden and great deceleration is reflected through the parameter $v_{d}$.

Moussa pointed out that the judgment conditions for DS (see (15)) proposed by Boccara et al. suggest that the careful driver has the same reaction time $(\tau=0)$ as the careless driver in [23], but the difference between them lies in different acceleration capability: the acceleration capability of careless drivers equals 2 while that of careful drivers equals 1 . In (17), the reaction time of the careless driver is $\tau \neq 0$ while that of the careful driver is $\tau=0$. They have the same acceleration capability.

In the two-lane model with an abandoned object (VATCA-AO and IoV-ATCA-AO), the sudden occurrence of a large abandoned object will seriously interfere with the driver's driving behavior, thus causing a traffic accident. In particular, if an abandoned object is ahead in the right lane, depending on whether the lane changing conditions satisfy, driving behavior will change to avoid hitting the abandoned object, thus causing the following two types of traffic accidents: (1) A Rear-End Collision Caused by the Abandoned Object Ahead. When lane changing conditions 
for the vehicle in the lane with the abandoned object ahead are not satisfied, the vehicle can only slow down to avoid hitting the abandoned object. If this deceleration is very great, a rear-end collision may result from the succeeding vehicle. (2) A collision of the Vehicle with the Abandoned Object. When the lane changing conditions are satisfied and an abandoned object is ahead in the right lane, the vehicle will change lanes to avoid hitting the abandoned object. In the case of a sudden occurrence of an abandoned object, if the succeeding vehicle slows down too late, the vehicle will hit it. For convenience, a rear-end collision caused by the abandoned object ahead is called a "type I" accident, and a collision of the vehicle with an abandoned object is called a "type II" accident in this article.

For corresponding judgment conditions for DS about type I accidents, limits of lane changes can be added on the basis of (17) proposed by Moussa, as shown below:

$$
\begin{aligned}
& \text { (i) } x(k, t)<x(k+1, t)<x_{\mathrm{ao}}<x(k+2, t) \\
& \text { (ii) } c(k, t)=0 \\
& \text { (iii) } c(k+1, t)=0 \\
& \text { (iv) } \tau v(k, t)>d(k, t)+v(k+1, t+1) \\
& \text { (v) } v(k+1, t)-v(k+1, t+1) \geq v_{d},
\end{aligned}
$$

where $c(k, t)$ denotes whether the lane changing conditions are satisfied for vehicle $k$ at the time step $t$ (1 means satisfied; 0 means dissatisfied). Condition (i) of (18) denotes that the location of the abandoned object is ahead of the preceding vehicle $k+1$ at the time step $t$. Conditions (ii) and (iii) of (18), respectively, denote that the lane changing conditions cannot be satisfied for vehicle $k$ and the preceding vehicle $k+1$ at the time step $t$. Conditions (iv) and (v) of (18) are identical with (17) proposed by Moussa.

Corresponding judgment conditions for DS about a type II accident are given in

$$
\begin{aligned}
& \text { (i) } x(k, t)<x(k+1, t)<x_{\mathrm{ao}}<x(k+2, t) \\
& \text { (ii) } c(k, t)=0 \\
& \text { (iii) } c(k+1, t)=1 \\
& \text { (iv) } \tau v(k, t)>d_{\mathrm{ao}}(k, t) \\
& \text { (v) } v(k+1, t)>0 .
\end{aligned}
$$

Condition (i) of (19) denotes that the location of the abandoned object is ahead of the preceding vehicle $k+1$. Condition (ii) of (19) denotes that vehicle $k$ does not change lanes at the time step $t$, while condition (iii) of (19) denotes that the preceding vehicle $k+1$ suddenly changes lanes at the time step $t$. Condition (iv) of (19) denotes that, in the reaction time $\tau$, if vehicle $k$ does not slow down, it will travel up to the location of the abandoned object and hit it. Condition (v) of (19) denotes that the velocity of the preceding vehicle $k+1$ at the time step $t$ is not equal to zero. According to condition (v), if $v(k+1, t)=0$, this means that the state of the preceding vehicle $k+1$ is parked at time step $t$, and the driver of vehicle $k$ will drive carefully and will not hit the abandoned object.

\section{Simulation Results and Discussion}

4.1. Configurations. We simulated a two-lane traffic model with the periodic boundary condition. The length of a cell is $7.5 \mathrm{~m}$, and the road length is $15 \mathrm{~km}$; thus, $L=2000$ cells. Vehicle density is defined as $\rho=N / 2 L$, where $N$ is vehicle count. The abandoned object is located in the middle in the right lane; that is, $x_{\mathrm{ao}}=1000$. The visual range of abandoned object $d_{\mathrm{v}}$ depends on the size of the abandoned object, and the influence of large abandoned objects on traffic accidents is studied in this article. In this study, $d_{\mathrm{v}}=10$; for $d_{\text {safe }}$, in some papers $d_{\text {safe }}=v_{\max }$. When the vehicle density is larger, the velocity can not reach $v_{\max }$. We used $d_{\text {safe }}=\min \left(v_{\text {succ }}(k, t)+\right.$ $\left.1, v_{\max }\right)$. The other parameters are given by the probability of the randomization $p=0.2$, the maximal velocity is $v_{\max }=5$, the first-warning threshold is $d_{\mathrm{v} 2 \mathrm{v}}^{1}=200$, the second-warning threshold is $d_{\mathrm{v} 2 \mathrm{v}}^{2}=20$, the delayed reaction time is $\tau=1$, and the deceleration limit is $v_{d}=2$. To reduce the influence of random factors, the values of 30 simulations are averaged in every density. The sampling step begins at the 50,000th time step and ends at the 150,000th time step.

For the study of the above two kinds of traffic accidents caused by an abandoned object, $p_{\mathrm{ac}}$ denotes the accident rate per time step for an accident to occur, which is defined as follows:

$$
p_{\mathrm{ac}}=p_{\mathrm{col}} \frac{1}{T} \sum_{t=1}^{T} \mathrm{DS}(t),
$$

where $p_{\text {col }}$ denotes the probability of the careless drivers and $T$ means the length of the sampling time step. For type I or type II accidents, there is only one vehicle in DS at time step $t$. DS $(t)$ denotes whether the corresponding judgment condition of DS for type I or type II accidents at time step $t$ is satisfied ( 1 means satisfied; 0 means dissatisfied). The value $p_{\text {col }}$ will be affected by different environments; therefore, $p_{\text {ac }} / p_{\text {col }}$ denotes the traffic accident rate [23].

4.2. Accident Rate Analysis. For V-ATCA-AO and IoVATCA-AO, DS judgment conditions for a type I accident use (18), while DS judgment conditions for a type II accident use (19). The type I accident rate curve for these two models is illustrated in Figure 4, and the type II accident rate curve for these two models is illustrated in Figure 5.

Simulation results demonstrate that when the vehicle density is great, the probability of the occurrence of type I and type II accidents is very low. Therefore, to ease comparison, the density used in Figures 4 and 5 coordinates only to 0.2. $\left[\rho_{\text {low_I }}^{\mathrm{V}}, \rho_{\text {high_I }}^{\mathrm{V}}\right]$ and $\left[\rho_{\text {low_I }}^{\mathrm{IoV}}, \rho_{\text {high_I }}^{\mathrm{IoV}}\right]$ denote the density intervals when a type I accident occurs in the two models, and $p_{\text {max_I }}^{\mathrm{V}}$ and $p_{\text {max_I }}^{\mathrm{IoV}}$ denote maximal accident rate. $\rho_{\text {max_I }}^{\mathrm{V}}$ and $\rho_{\text {max_I }}^{\text {IoV_I }}$, respectively, denote the density when $p_{\text {max_I }}^{\mathrm{V}}$ and $p_{\text {max_I }}^{\mathrm{IoV}}$ occur; $\left[\rho_{\text {low_II }}^{\mathrm{V}}, \rho_{\text {high_II }}^{\mathrm{V}}\right]$ and $\left[\rho_{\text {low_II }}^{\mathrm{IoV}}, \rho_{\text {high_II }}^{\mathrm{IoV}}\right]$ denote the density intervals when a type II accident occurs, and $p_{\text {max_II }}^{\mathrm{V}}$ and $p_{\text {max_II }}^{\text {IoV }}$ denote the maximal accident rate; $\rho_{\text {max_II }}^{\mathrm{V}}$ and $\rho_{\text {max_II }}^{\text {IoV }}$, respectively, denote the density when $p_{\text {max_II }}^{\mathrm{V}}$ and $p_{\max \_ \text {II }}^{\mathrm{IoV}}$ occur. 


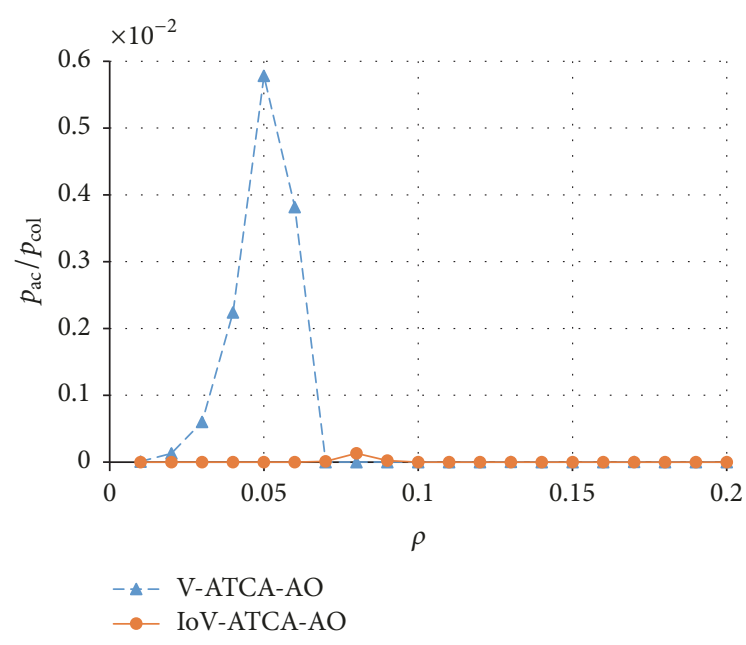

FIGURE 4: Type I accident rate in two kinds of model.

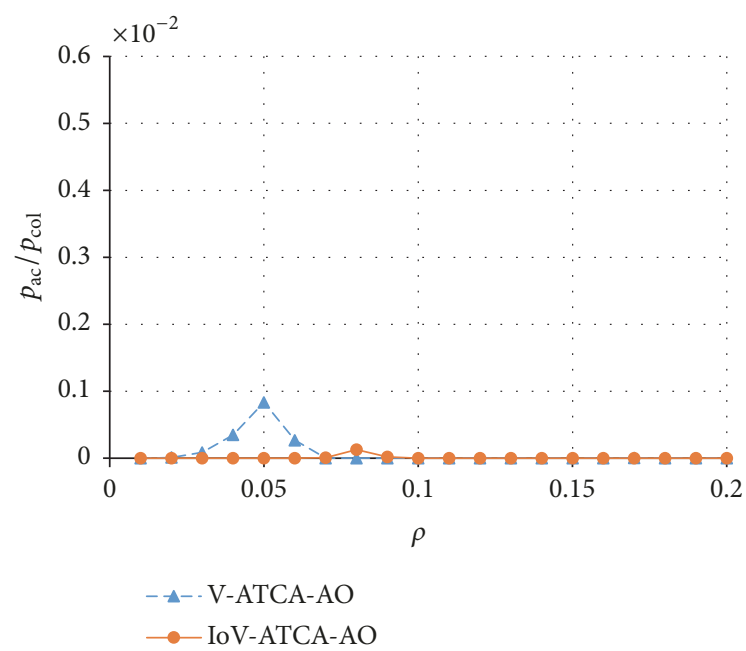

FIgURE 5: Type II accident rate in two kinds of model.

In Figure 4 , in the visual environment, $\left[\rho_{\text {low_I }}^{\mathrm{V}}, \rho_{\text {high_I }}^{\mathrm{V}}\right]$ denotes the density interval when a type I accident occurs, and the value is $[0.01,0.07]$, and, accordingly, $\rho_{\max I}^{\mathrm{V}}=0.05$ and $p_{\text {max }}^{\mathrm{V}}=0.578 \times 10^{-2}$. In the Internet-of-Vehicles environment, $\left[\rho_{\text {low_I }}^{\text {IoV }}, \rho_{\text {high_I }}^{\text {IoV }}\right]$ denotes the density interval when a type I accident occurs, and the value is [0.07, 0.09], and, accordingly, $\rho_{\text {max_I }}^{\mathrm{IoV}}=0.08$ and $p_{\text {max_I }}^{\mathrm{IoV}}=0.013 \times 10^{-2}$.

In Figure 5 , in the visual environment, $\left[\rho_{\text {low_II }}^{\mathrm{V}}, \rho_{\text {high_II }}^{\mathrm{V}}\right]$ denotes the density interval when a type II accident occurs, and the value is $[0.02,0.07]$, and, accordingly, $\rho_{\max I I}^{\mathrm{V}}=$ 0.05 and $p_{\text {max_II }}^{\mathrm{V}}=0.084 \times 10^{-2}$. In Internet-of-Vehicles environment, [ $\left.\rho_{\text {low_II }}^{\mathrm{IoV}}, \rho_{\text {high_II }}^{\mathrm{IoV}}\right]$ denotes the density interval when a type II accident occurs, and the value is $[0.07,0.09]$, and, accordingly, $\rho_{\max \text { II }}^{\mathrm{IoV}}=0.08, p_{\max \text { II }}^{\mathrm{IoV}}=0.013 \times 10^{-2}$.

From Figures 4 and 5, the accident rates of the two models can be obtained.

(1) In V-ATCA-AO, [0.01, 0.07] and [0.02, 0.07] denote the density intervals when two types of accidents occur; the numbers are basically the same. $\rho_{\max \mathrm{I}}^{\mathrm{V}}$ is the same as $\rho_{\max \_\mathrm{II}}^{\mathrm{V}}$; both are 0.05 ; the accident rate curve is different in scale, but the shape is similar. This shows that, for the visual-based avoidance pattern with abandoned object, there are similar features between two types of accidents and density.

(2) In IoV-ATCA-AO, density intervals are the same when the two types of accidents occur and both are [0.07, 0.09]; $\rho_{\text {max_I }}^{\mathrm{IoV}}$ is the same as $\rho_{\text {max_II }}^{\mathrm{IoV}}$ and both are 0.08 ; not only the accident rate curve but also the shape is similar. This shows that, with the Internet-of-Vehicles-based avoidance pattern with abandoned object, there are similar features between two types of accidents and density.

(3) $\rho_{\text {high_I }}^{\mathrm{V}}, \rho_{\text {high_II }}^{\mathrm{V}}, \rho_{\text {high_I }}^{\mathrm{IoV}}$, and $\rho_{\text {high_II }}^{\mathrm{IoV}}$ are all smaller (less than 0.1 . This indicates that both types of accidents occur when the density is smaller, but when the density is larger, both types of accident will not occur. The results are different from the rear-end collision rate curve without the abandoned object in a single lane [23] according to (17), in Figure 11 of [23], indicating that a rear-end collision will still occur when the density is larger.

(4) In V-ATCA-AO, $p_{\text {max_I }}^{\mathrm{V}} / p_{\text {max_II }}^{\mathrm{V}}=6.9$. This indicates that, with the visual-based avoidance pattern with an abandoned object, a type I accident is more likely to occur than a type II accident.

(5) In IoV-ATCA-AO, $p_{\text {max_I }}^{\mathrm{IoV}} / p_{\text {max_II }}^{\mathrm{IoV}}=1.0$. This indicates that type I accidents and type II accidents occur with the same probability.

(6) For type I accidents, $\rho_{\text {low_I }}^{\mathrm{IoV}}>\rho_{\text {low_I }}^{\mathrm{V}}$ and $\rho_{\text {high_I }}^{\mathrm{IoV}}>\rho_{\text {high_I }}^{\mathrm{V}}$; for type II accidents, $\rho_{\text {low_II }}^{\text {IoV }}>\rho_{\text {low_II }}^{\mathrm{V}}$ and $\rho_{\text {high_II }}^{\text {IoV }}>\rho_{\text {high_II }}^{\mathrm{V}}$. This indicates that, compared to the visual-based avoidance pattern with abandoned object, for the Internet-of-Vehiclesbased avoidance pattern with abandoned object the density intervals of the occurrence of both types of accidents are much higher.

(7) For the type I accident, $p_{\text {max_I }}^{\mathrm{V}} / p_{\text {max_I }}^{\mathrm{IoV}}=44.5$; for the type II accident, $p_{\text {max_II }}^{\mathrm{V}} / p_{\max \text { II }}^{\text {IoV }}=6.5$. This indicates that, compared to the visual-based avoidance pattern with abandoned object, the Internet-of-Vehicles-based avoidance pattern with abandoned object can greatly reduce the rate of the two types of accidents.

Based on characteristics of the two types of accidents in two models, and the effect of early warning of an abandoned object on traffic accidents, the Internet-of-Vehicles-based avoidance pattern with abandoned object can effectively reduce the rate of the two types of accidents.

4.3. Effect Analysis of Accident Rate with Spatiotemporal Characteristics of Traffic Flow. In this section, the causes of accident rate characteristics are analyzed by spatiotemporal diagrams. Spatiotemporal diagrams of several typical density in V-ATCA-AO are shown in Figure 6: $\rho_{\text {low-I }}^{\mathrm{V}}, \rho_{\max -\mathrm{I}}^{\mathrm{V}}-1, \rho_{\max -\mathrm{I}}^{\mathrm{V}}$, $\rho_{\text {max }-\mathrm{I}}^{\mathrm{V}}+1$, and $\rho_{\text {high-I }}^{\mathrm{V}}$, such as $\rho=\{0.01,0.04,0.05,0.06,0.07\}$. Spatiotemporal diagrams of several typical densities in IoVATCA-AO are shown in Figure $7: \rho_{\text {low II }}^{\text {IoV }}, \rho_{\text {max II }}^{\text {IoV }}-1, \rho_{\text {max I }}^{\text {IoV }}$, $\rho_{\text {max_I }}^{\text {IoV }}+1$, and $\rho_{\text {high } \_ \text {I }}^{\text {IoV }}$, for $\rho_{\text {low_I }}^{\text {IoV }}=\rho_{\text {max_I }}^{\text {IoV }}-1$ and $\rho_{\text {high_I }}^{\text {IoV }}=$ $\rho_{\text {max_I }}^{\text {IoV }}+1$, so $\rho=\{0.07,0.08,0.09\}$. Because traffic accidents 


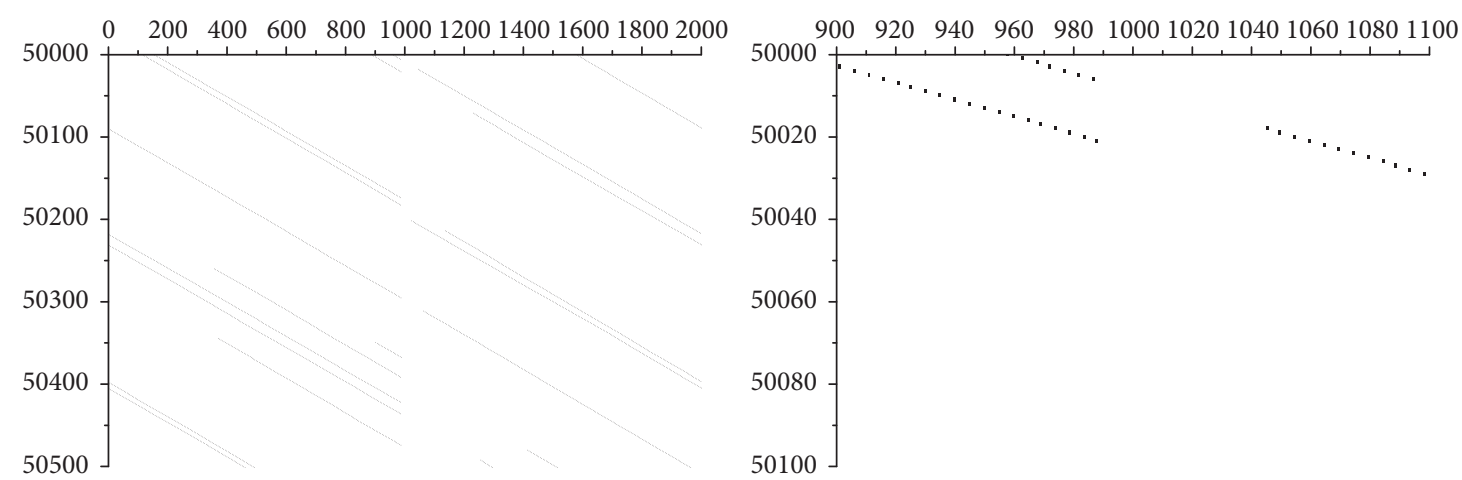

(a) $\rho=0.01$

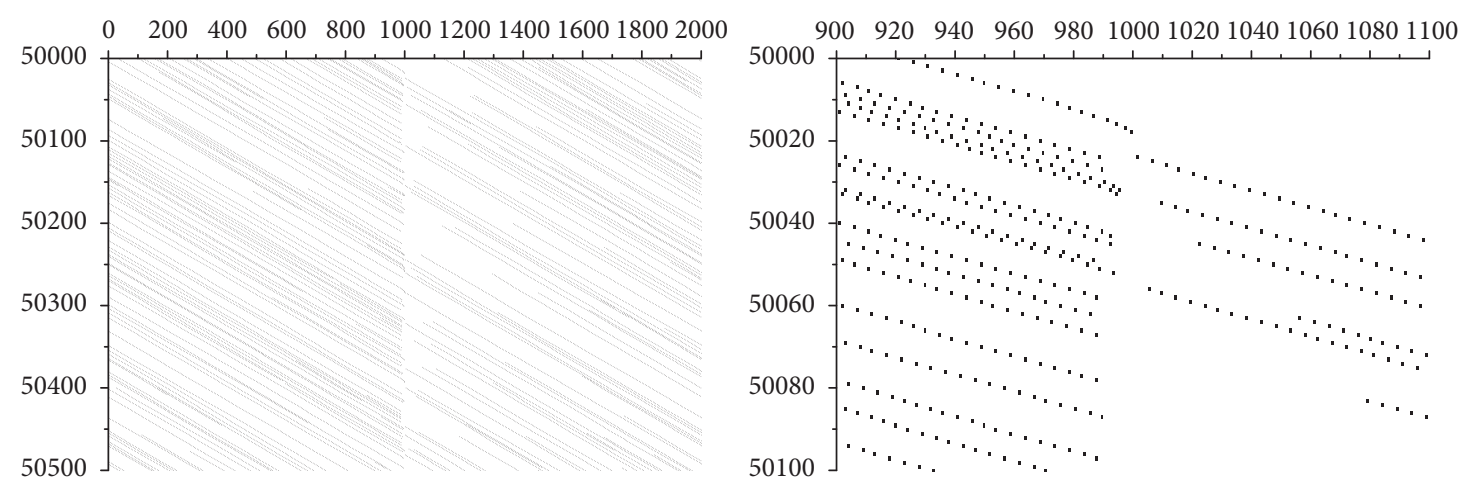

(b) $\rho=0.04$

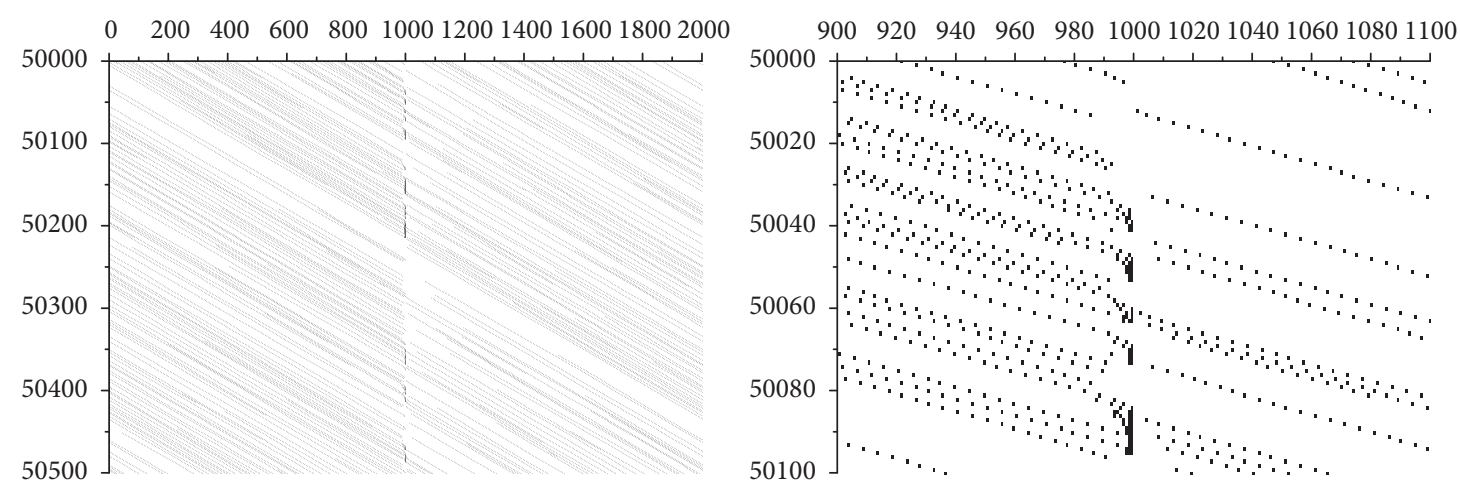

(c) $\rho=0.05$
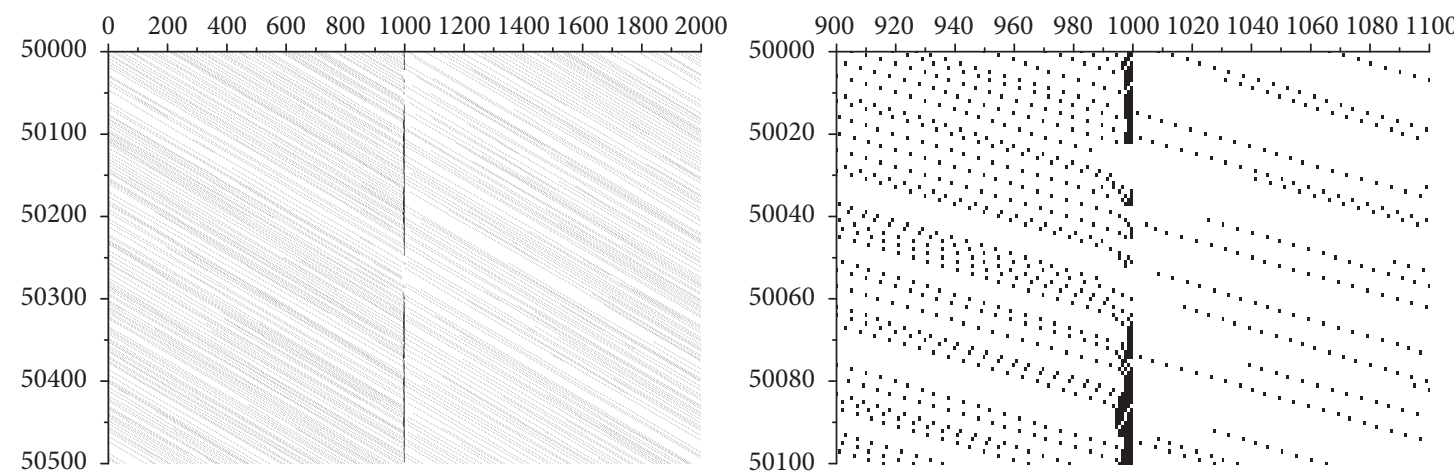

(d) $\rho=0.06$

FIgure 6: Continued. 

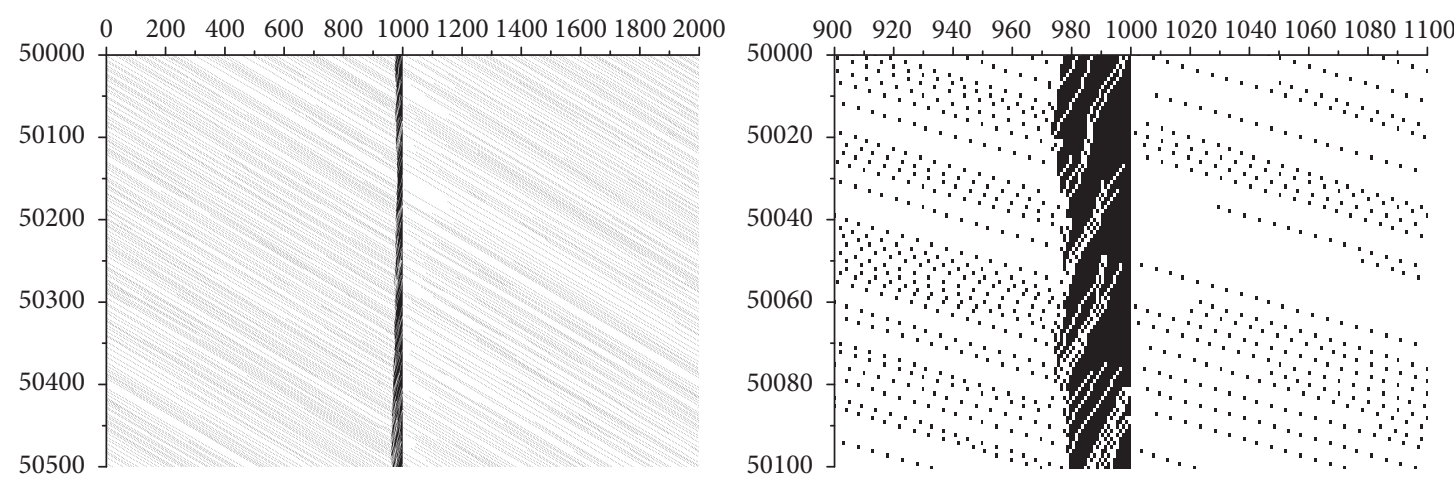

(e) $\rho=0.07$

FIGURE 6: Spatiotemporal diagrams of the right lane in V-ATCA-AO.

with abandoned object in the right lane are analyzed in this study, only spatiotemporal diagrams of the right lane are shown in Figures 6 and 7. To describe the spatiotemporal characteristics of the abandoned object nearby in detail, local spatiotemporal diagrams of abandoned objects nearby are given on the right side of each spatiotemporal diagram.

In V-ATCA-AO, when the driver in the right lane sees the abandoned object for the first time in a certain time step, if the lane changing incentive is satisfied $\left(x(k, t)<x_{\mathrm{ao}}<x(k+1, t)\right.$ and $\left.d_{\mathrm{ao}}(k, t)<d_{\mathrm{v}}\right)$ but the safety criterion $\left(d_{\text {succ }}(k, t)>d_{\text {safe }}\right)$ is not, the vehicle with higher speed at the next time step $t+1$ may greatly decelerate to avoid hitting the abandoned object. A type I accident may be caused at the next time step $t+1$ if the succeeding vehicle is close; if no type I accident occurs at the next time step $t+1$ because the vehicle succeeded in slowing after great deceleration, at the following time step, the possibility of great deceleration once again is very small and there is a little probability of a type I accident.

Thus, type I accidents mainly occur when the driver in the right lane sees the abandoned object for the first time; if the condition does not satisfy the safety criterion for a lane change, the situation is called DS1. The occurrence of DS1 is the premise of a type I accident. The greater the probability of DS1, the greater the probability of a type I accident.

In DS1, factors that determine whether a type I accident will occur include whether there is great deceleration of the vehicle and the following distance of the succeeding vehicle $d(k-1, t)$. The greater the probability of great deceleration of the vehicle and the closer the following distance of the succeeding vehicle, the greater the probability of a type I accident. If the vehicle decelerates greatly, the gap $d_{\mathrm{ao}}(k, t)$ between the vehicle and the abandoned object is very important; when the driver sees the abandoned object for the first time, the smaller the gap, the greater the probability for great deceleration.

In V-ATCA-AO, the visual distance of the abandoned object is $d_{\mathrm{v}}=10$, and the location of the abandoned object is $x_{\mathrm{ao}}=1000$, so the location interval of the abandoned object is $[990,999]$ that can be seen by the driver. From Figure 6, spatiotemporal diagrams of V-ATCA-AO can be seen.
(1) When $\rho=\rho_{\text {low I I }}^{\mathrm{V}}\left(\rho_{\text {low I }}^{\mathrm{V}}=0.01\right)$, as shown in Figure 6(a), because of the smaller density and the bigger gap $d_{\text {succ }}(k, t)$ between the vehicle and the succeeding vehicle in the neighboring lane, when the driver sees the abandoned object, the safety conditions for lane changing will be more easily satisfied, and in this case the vehicle will be able to change a lane earlier, and the probability of DS1 is smaller. If safety conditions are not satisfied, with the occurrence of DS1, in this case, (a) an earlier lane change of the preceding vehicle makes the gap $d_{\mathrm{ao}}(k, t)$ between the vehicle and the abandoned object much bigger when the driver sees the abandoned object for the first time and, (b) because of the very small density, the following distance of the succeeding vehicle is much bigger. Therefore, the probability of the occurrence of a type I accident is very low when the density is $\rho=\rho_{\text {low }}^{\mathrm{V}}$.

(2) In the density interval $\left[\rho_{\text {low I I }}^{\mathrm{V}}, \rho_{\text {max I }}^{\mathrm{V}}\right]$, that is $[0.01,0.05]$, as shown in Figures 6(a)-6(c), with the increase of density, the gap $d_{\text {succ }}(k, t)$ between the vehicle and the successor in the neighboring lane gradually decreases. When the driver sees the abandoned object, the safety conditions for a lane change have gradually decreased. The enhanced difficulty of a lane change increases the probability of DS1. In the visible range of the abandoned object, when the driver sees the abandoned object for the first time: (a) the gap $d_{\mathrm{ao}}(k, t)$ between the vehicle and the abandoned object has gradually decreased with the increase of density, and (b) the following distance of the succeeding vehicle has gradually decreased with the increase of density. As a result, the probability of DS1 has gradually increased. The probability of great deceleration has gradually increased, and the following distance has gradually decreased; therefore the probability of a type I accident rate has gradually increased.

(3) In the density interval $\left[\rho_{\text {max_I }}^{\mathrm{V}}, \rho_{\text {high_I }}^{\mathrm{V}}\right]$, that is $[0.05,0.07]$, as shown in Figures $6(\mathrm{c})-6(\mathrm{e})$, a traffic jam occurs in front of the abandoned object. This traffic jam is not a dense jam, but when a vehicle in the traffic jam changes a lane, the succeeding vehicle moves forward with low velocity, thereby producing a moving blank and a backward movement in the traffic jam. The traffic jam forms because 

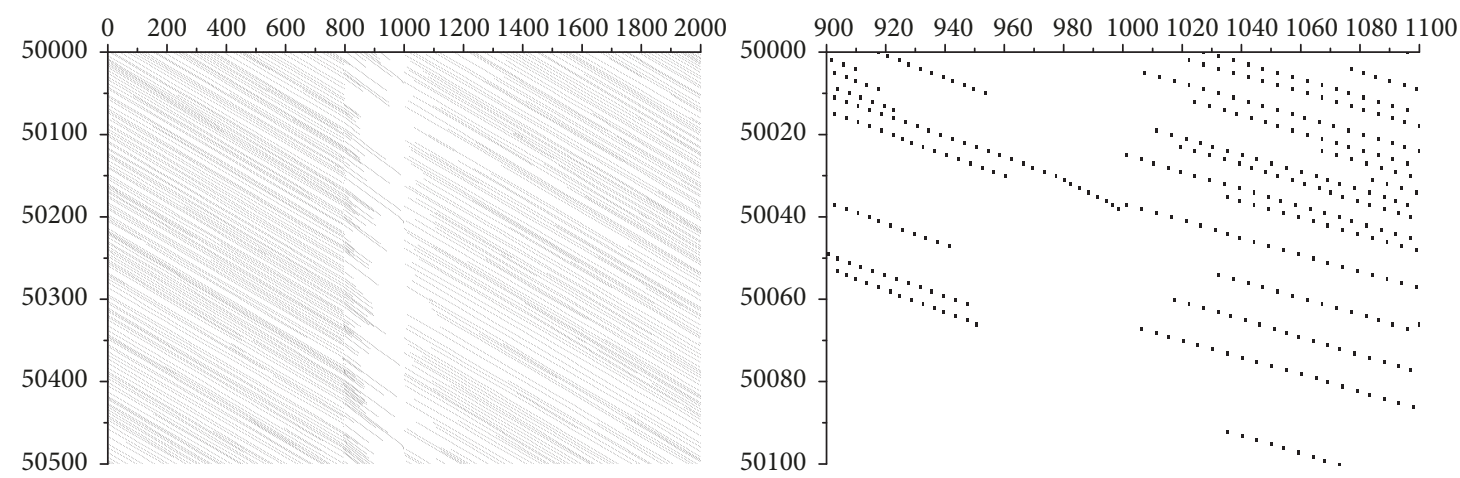

(a) $\rho=0.07$
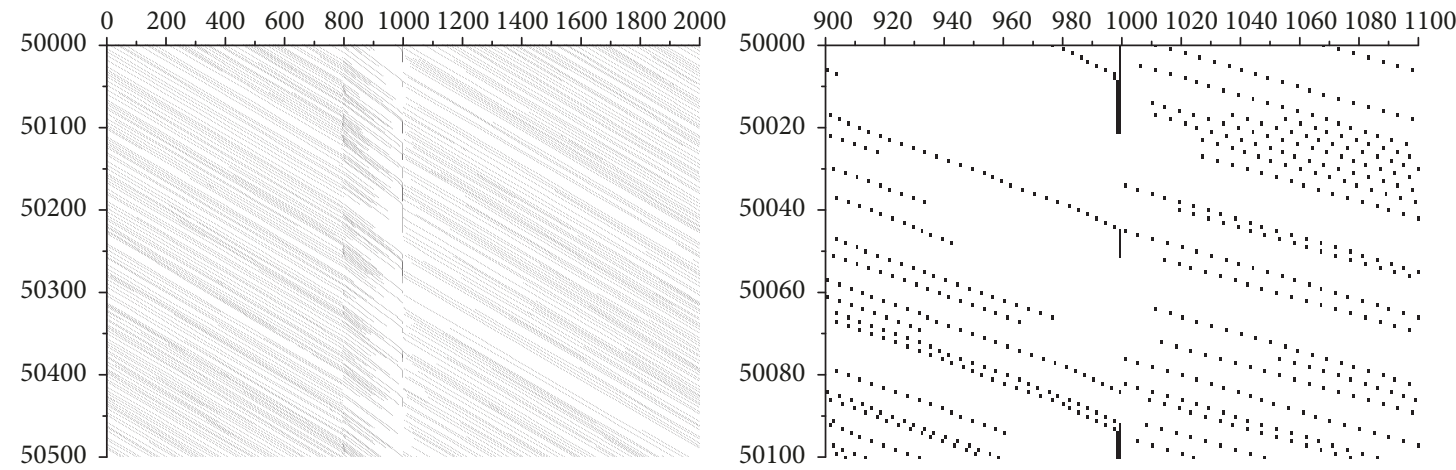

(b) $\rho=0.08$
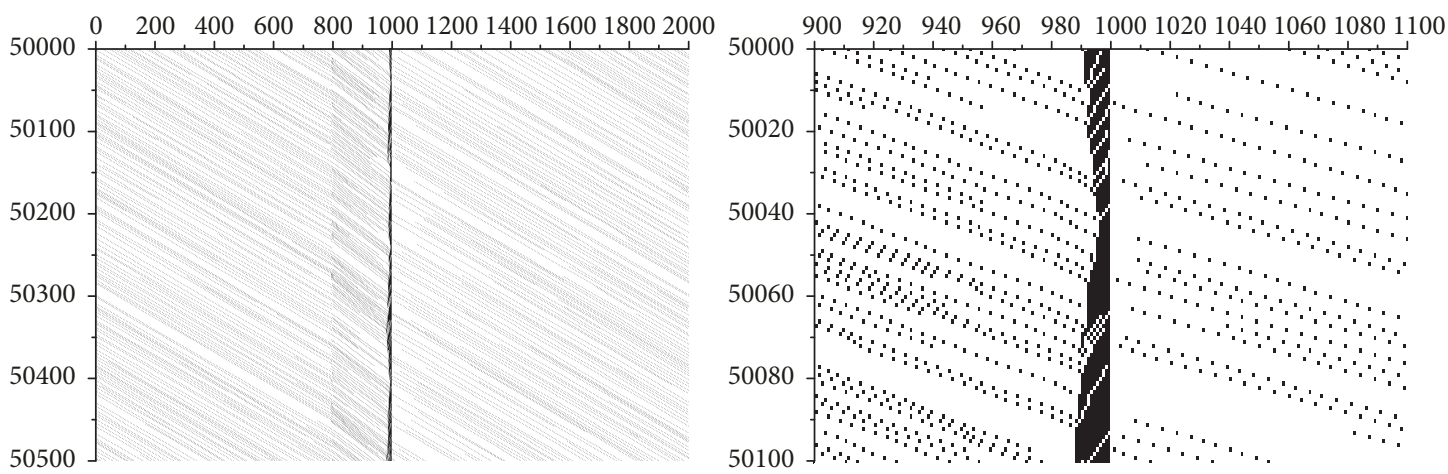

(c) $\rho=0.09$

FIgURE 7: Spatiotemporal diagrams of the right lane in IoV-ATCA-AO.

when a vehicle sees the abandoned object or another vehicle stopping in front of the abandoned object and the safety conditions are not satisfied, the vehicle decelerates and stops in front of the abandoned object. Obviously, the greater the density, the more easily the traffic jam forms. The greater the density, the less easily the traffic jam dissipates. Thus, the width of the traffic jam grows and its duration is longer. In the density interval $\left[\rho_{\text {max I }}^{\mathrm{V}}, \rho_{\text {high } \_}^{\mathrm{V}}\right]$, with the increase of density, the "stability" of the traffic jam is gradually enhanced. When $\rho=\rho_{\text {max I }}^{\mathrm{V}}$, as shown in Figure 6(c), the "stability" of the traffic jam is very poor with obvious features "disappearing and reappearing": the traffic jam occurs and disappears dynamically with small width and short duration. When $\rho=\rho_{\text {high_I }}^{\mathrm{V}}$, as shown in Figure 6(e), the traffic jam reaches a completely stable state: a traffic jam with persistence and fixed width. Vehicles are stopped or move forward in a slow state in the traffic jam. Therefore, great deceleration in front of the abandoned object will not occur, and type I accidents will not occur during a traffic jam. The dynamic process of forming a traffic jam is a rapid deceleration without lane changing in front of the abandoned object. In the density interval of the traffic jam, the bigger the probability of a traffic jam forming dynamically, the less stable the traffic jam, and the higher the rate for a type I accident. In the density interval $\left[\rho_{\text {max_I }}^{\mathrm{V}}, \rho_{\text {high_I }}^{\mathrm{V}}\right]$, when $\rho=\rho_{\text {max_I }}^{\mathrm{V}}$, the traffic jam is least stable, so the corresponding rate of type I accident is 
highest. With the increase of density, the "stability" of the traffic jam increases, so the corresponding rate of type I accident decreases.

(4) When $\rho \geq \rho_{\text {high_I }}^{\mathrm{V}}$, the traffic jam comes into a completely stable state, and therefore a type I accident will not occur. Initial density $\rho_{\text {high_I }}^{\mathrm{V}}$ is smaller when the traffic jam is produced in a completely stable state, so a type I accident occurs when the density is smaller but will not occur when the density is larger.

Characteristics of spatiotemporal diagrams above have explained well the features of type I accident rate in V-ATCA$\mathrm{AO}$ in Figure 4.

In IoV-ATCA-AO, the early warning for an abandoned object allows the driver to generate an incentive to change a lane in advance and to make an earlier lane change. In addition, according to the warning principle proposed in Section 2.3, when vehicles in the right lane enter into the general warning zone and the emergency warning zone, the highest velocity is gradually reduced. The earlier lane change and deceleration behavior in advance change spatiotemporal characteristics in front of the abandoned object so as to bring different characteristics of the type I accident rate in the two models mentioned in Figure 4. As shown in Figures 6 and 7, the difference of the spatiotemporal diagrams in two models can explain well the difference of accident rate characteristics.

(1) In IoV-ATCA-AO, when $\rho=\rho_{\text {low I-I }}^{\text {Iov }}$, the spatiotemporal characteristics in front of the abandoned object in Figure 7(a) are similar to those in Figure 6(a) in V-ATCA-AO when $\rho=$ $\rho_{\text {low_I}}^{\mathrm{V}}$. Thus, the accident rates of both densities of the two models are very low. However, the value of $\rho_{\text {low_I }}^{\text {Iov }}(0.07)$ is much greater than the value of $\rho_{\text {low_I }}^{\mathrm{V}}(0.01)$ because the vehicle can achieve earlier lane change with IoV-ATCA-AO.

(2) In IoV-ATCA-AO, when $\rho=\rho_{\max \text { II }}^{\text {IoV }}$, the spatiotemporal characteristics in front of the abandoned object in Figure 7(b) are similar to those in Figure 6(c), in V-ATCA-AO when $\rho=\rho_{\text {max } \_ \text {I. }}^{\mathrm{V}}$. But, for a type I accident, $p_{\text {max } \mathrm{I}}^{\mathrm{V}} / p_{\text {max } \_ \text {I }}^{\mathrm{IoV}}=$ 44.5 , because in IoV-ATCA-AO vehicles can decelerate in advance. In V-ATCA-AO, if the driver in the right lane does not see the abandoned object ahead and is driving at maximum velocity $v_{\max }$, when the abandoned object first appears in the front and he cannot change a lane, maximum velocity will be set to $v_{\max }-2$. If the velocity is higher, deceleration will be greater and the probability of producing a great deceleration is much bigger. But, in IoV-ATCA-AO, when the driver in the right lane sees the abandoned object ahead, the vehicle is in an emergency warning zone with the top velocity $v_{\max }-2$ already. In this case, the probability of a great deceleration for the vehicle is much smaller. Compared with the visual-based avoidance pattern with abandoned object, the Internet-of-Vehicles-based avoidance pattern with abandoned object can greatly reduce the accident rate.

(3) In Figure 4, type I accident rate in IoV-ATCA-AO is larger than that in V-ATCA-AO in the density interval $[0.07,0.09]$. In IoV-ATCA-AO, the early warning for an abandoned object allows the vehicle close to the abandoned object to generate an incentive to change lane in advance. However, whether the lane change is successful depends on the safety criterion $d_{\text {succ }}(k, t)>d_{\text {safe }}$. Therefore, from the spatiotemporal diagram shown in Figure 7, in the density interval $[0.07,0.09]$, with the increase of density, it is more and more difficult for the vehicle to change lane near the abandoned object in the right lane. Thus, a traffic jam is gradually generated in front of the abandoned object. When $\rho=0.07$, the jam is almost absent; when $\rho=0.08$, the "stability" of the traffic jam is very poor with the feature of "disappearing and reappearing"; when $\rho=0.09$, the traffic jam reaches a completely stable state. According to the characteristics between accident rate and traffic jam (in the density interval of the traffic jam, the bigger the probability of a traffic jam forming dynamically, the less stable the traffic jam, and the higher the rate for a type I accident), in the density interval $[0.07,0.09]$, type I accident occurs in IoVATCA-AO and the accident rate reaches the maximum when $\rho=0.08$. However, in V-ATCA-AO, since there is no early warning, the vehicle close to the abandoned object in the right lane can only generate an incentive to change lane when the abandoned object is found in the visual range. Therefore, in the same density, the traffic jam in V-ATCA-AO occurs easier compared with IoV-ATCA-AO. The above phenomenon can be shown in the spatiotemporal diagrams in Figures 6 and 7. In Figure 6(e), when $\rho=0.07$, the traffic jam reaches a completely stable state, so no type I accident occurs in VATCA-AO. We further analyzed the spatiotemporal diagrams when $\rho=0.08$ and $\rho=0.09$ in V-ATCA-AO. The results show that, with the increase of density, characteristics of spatiotemporal diagrams remain unchanged, and the traffic jam is still in a stable state with the increase of length. So, type I accident also will not occur when $\rho=0.08$ and $\rho=$ 0.09 . Because characteristics of spatiotemporal diagrams are similar when $\rho=0.08$ and $\rho=0.09$ to that when $\rho=0.07$, the spatiotemporal diagrams are not shown when $\rho=0.08$ and $\rho=0.09$. Therefore, in the density interval $[0.07,0.09]$, the type I accident rate in IoV-ATCA-AO is larger than that in V-ATCA-AO.

4.4. Effect Analysis of Accident Rate by Warning Threshold. In the above analysis of accident rate, the first-warning threshold of the gap between the vehicle terminal and the abandoned object is $d_{\mathrm{v} 2 \mathrm{v}}^{1}=200 . d_{\mathrm{v} 2 \mathrm{v}}^{1}$ is bigger, suggesting that there will be an earlier warning. A type I accident is taken as an example in this section to analyze the effect of various values of $d_{\mathrm{v} 2 \mathrm{v}}^{1}$ on the accident rate. Five values of $d_{\mathrm{v} 2 \mathrm{v}}^{1}(50,100,200,100$, and 200 ) for the type I accident rate are given in IoV-ATCA-AO, as shown in Figure 8.

Figure 8 shows the following: (1) For the five values of $d_{\mathrm{v} 2 \mathrm{v}}^{1}$, the curve shape of the accident rate is similar: the difference in the width of the density interval [ $\left.\rho_{\text {low_I }}^{\mathrm{IoV}}, \rho_{\text {high_I }}^{\mathrm{IoV}}\right]$ of occurrence of accidents is much smaller, as is the maximal accident rate $p_{\max \mathrm{I}}^{\mathrm{IoV}}$. (2) With the increase of $d_{\mathrm{v} 2 \mathrm{v}}^{1}$, the density interval $\left[\rho_{\text {low }}^{\mathrm{IOV}} \mathrm{I}, \rho_{\text {high_I }}^{\mathrm{IoV}}\right]$ of the occurrence of accidents gradually moves to the right, and the density $\rho_{\text {max_I }}^{\mathrm{IoV}}$ for the maximal accident rate gradually also moves to the right. This shows that the earlier the warning is, the higher the density interval is when an accident occurs. The reason is as follows. 


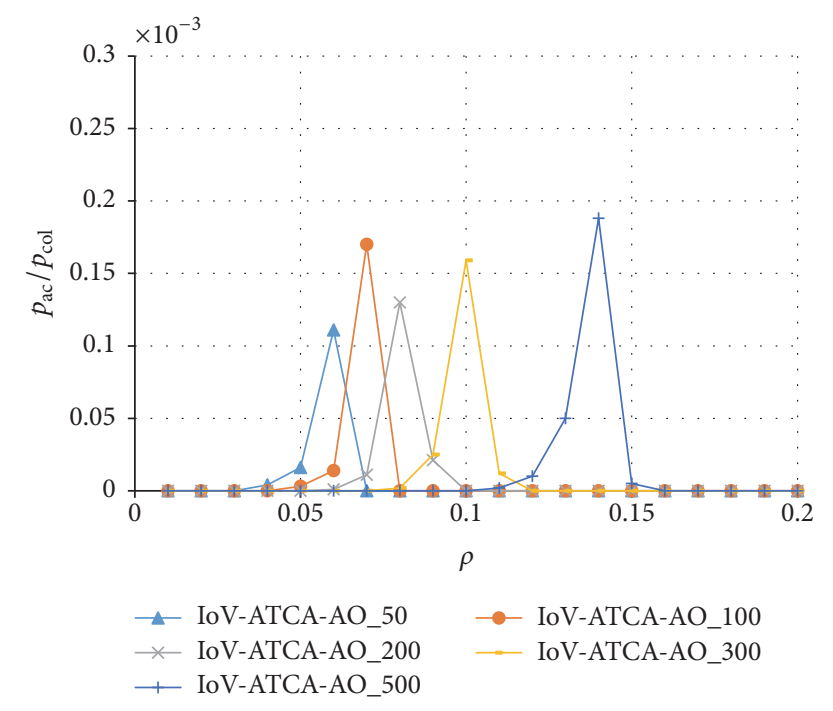

FIGURE 8: Effect of various $d_{\mathrm{v} 2 \mathrm{v}}^{1}$ on accident rate in IoV-ATCA-AO.

When the vehicle enters the warning area, the vehicle in the right lane generates the incentive to change lane, and, in next time steps, once the safety criterion is satisfied, the vehicle will change to the left lane. If the safety criterion is always not satisfied, the vehicle will stop in front of the abandoned object and may cause the traffic accident (type I). And, from spatiotemporal diagram, a traffic jam occurs in front of the abandoned object. Therefore, the vehicle in the right lane will have more time to change lane with larger warning threshold and the density for the traffic jam generated in front of the abandoned object is higher, which is the reason for the above conclusion (2). Although the density increases when the traffic jam is generated in front of the abandoned object due to the increase of the warning threshold, the local spatiotemporal characteristics in front of the abandoned object is similar, which is the reason for the above conclusion (1). The spatiotemporal diagram of the right lane is shown in Figure $7(\mathrm{~b})$ when $d_{\mathrm{v} 2 \mathrm{v}}^{1}=200$ and $\rho_{\max \text { I }}^{\text {Iov }}=0.8$. In order to illustrate the similarity, the spatiotemporal diagrams of the right lane for other $d_{\mathrm{v} 2 \mathrm{v}}^{1}$ and $\rho_{\text {max_I }}^{\mathrm{IoV}}$ are shown in Figure 9, which show the local spatiotemporal characteristics of the traffic jam in front of the abandoned object are similar to that in Figure 7(b).

For each density, there is a warning threshold interval $\left[d_{\mathrm{v} 2 \mathrm{v}}^{\text {low }}, d_{\mathrm{v} 2 \mathrm{v}}^{\text {high }}\right]$ for accidents: accidents will occur when the warning threshold $d_{\mathrm{v} 2 \mathrm{v}}^{1}$ is located in the area; otherwise, accidents will not occur. For example, in Figure 8, when $\rho=$ 0.09 , accident rate is greater than 0 for $d_{\mathrm{v} 2 \mathrm{v}}^{1}=\{200,300\}$, while accident rate is 0 for $d_{\mathrm{v} 2 \mathrm{v}}^{1}=\{50,100,500\}$. The warning threshold interval can be obtained as follows. For each density, we simulated with different $d_{\mathrm{v} 2 \mathrm{v}}^{1}$, which starts from 50 and increases by 10 every trial. And the warning threshold range can be obtained if the accident rate is not zero, as shown in Table 1. It is important for traffic management departments to set an appropriate warning threshold $d_{\mathrm{wt}}$. The setting method is that, for each density, $d_{\mathrm{wt}}>d_{\mathrm{v} 2 \mathrm{v}}^{\text {high }}$, as shown
TABLE 1: Warning threshold interval $\left[d_{\mathrm{v} 2 \mathrm{v}}^{\text {low }}, d_{\mathrm{v} 2 \mathrm{v}}^{\text {high }}\right]$ for traffic accidents and appropriate warning threshold $d_{\mathrm{wt}}$ in different density.

\begin{tabular}{lcc}
\hline$\rho$ & {$\left[d_{\mathrm{v} 2 \mathrm{v}}^{\text {low }}, d_{\mathrm{v} 2 \mathrm{v}}^{\text {high }}\right]$} & $d_{\mathrm{wt}}$ \\
\hline $0.01-0.04$ & - & 50 \\
0.05 & {$[50,60]$} & 70 \\
0.06 & {$[50,100]$} & 110 \\
0.07 & {$[80,200]$} & 210 \\
0.08 & {$[140,270]$} & 280 \\
0.09 & {$[200,320]$} & 330 \\
0.1 & {$[260,390]$} & 400 \\
0.11 & {$[310,440]$} & 450 \\
0.12 & {$[360,500]$} & 510 \\
0.13 & {$[410,550]$} & 560 \\
0.14 & {$[460,590]$} & 600 \\
0.15 & {$[510,640]$} & 650 \\
0.16 & {$[560,690]$} & 700 \\
0.17 & {$[600,740]$} & 750 \\
0.18 & {$[650,790]$} & 800 \\
0.19 & {$[700,830]$} & 840 \\
0.2 & {$[750,890]$} & 900 \\
\hline
\end{tabular}

in Table 1. Further, the appropriate warning thresholds of different density are given in Figure 10.

\section{Conclusion}

On the highway, the sudden appearance of an abandoned object will seriously interfere with the driver's driving behavior and can cause a traffic accident such as a rearend collision. Considering the influence of an abandoned object on driving behavior, a Visual-based Asymmetric Twolane Cellular Automata model with Abandoned Object (VATCA-AO) and an Internet-of-Vehicles-based Asymmetric Two-lane Cellular Automata model with Abandoned Object (IoV-ATCA-AO) are both proposed. Two types of traffic accidents caused by abandoned objects are analyzed: a rear-end collision caused by the abandoned object ahead (type I) and a collision of the vehicle with the abandoned object (type II). Corresponding accident judgment criterions are also proposed. On this basis, through spatiotemporal characteristics of traffic flow by simulation, accident rates of two models are discussed. Simulation results show the following: (1) Two types of accidents occur when density is smaller while two types of accident will not occur when the density is larger. The results are different from the rear-end collision accident rate curve without an abandoned object in a single lane, based on the literature (Moussa, 2003). (2) In the visual-based avoidance pattern with an abandoned object, a type I accident is likelier to occur than a type II. (3) In the Internet-of-Vehicles-based avoidance pattern with an abandoned object, type I accidents and type II accidents occur with the same probability. (4) Compared with the visual-based avoidance pattern with an abandoned object, in the Internet-of-Vehicles-based avoidance pattern with an abandoned object, density intervals of the occurrence of 


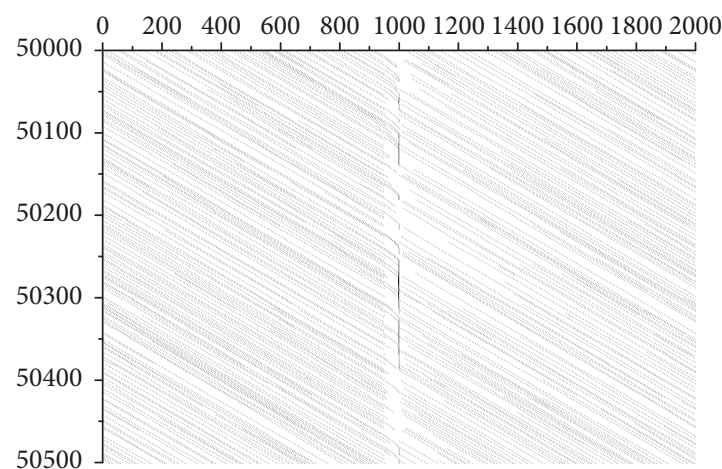

(a) $d_{\mathrm{v} 2 \mathrm{v}}^{1}=50 ; \rho_{\text {max } \mathrm{II}}^{\mathrm{IoV}}=0.06$

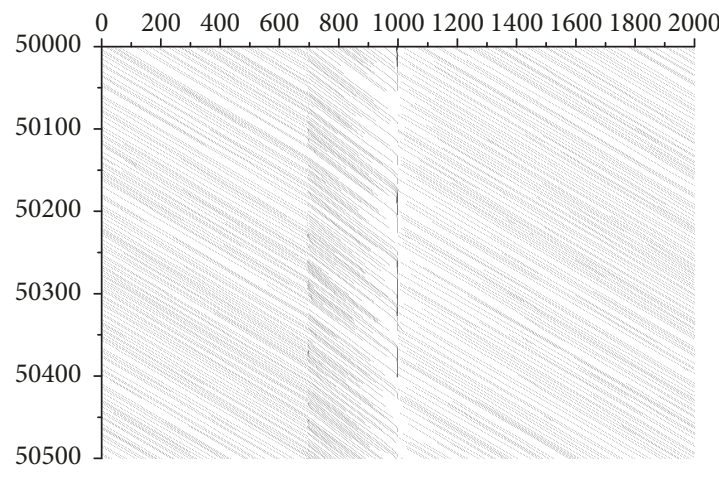

(c) $d_{\mathrm{v} 2 \mathrm{v}}^{1}=300 ; \rho_{\max \_\mathrm{I}}^{\mathrm{IoV}}=0.1$

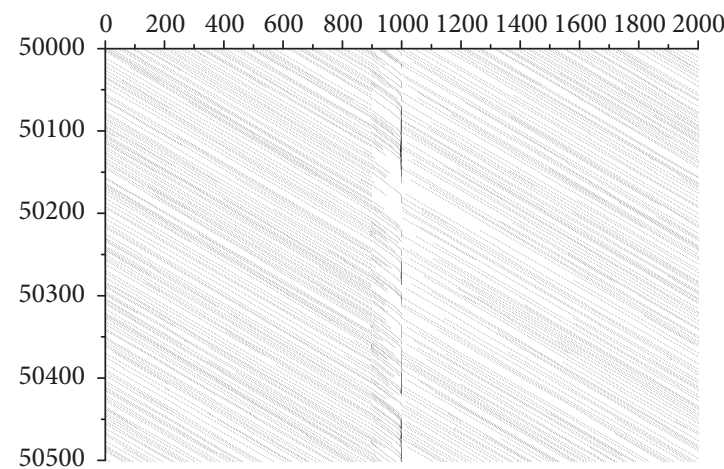

(b) $d_{\mathrm{v} 2 \mathrm{v}}^{1}=100 ; \rho_{\text {max II }}^{\mathrm{IoV}}=0.07$

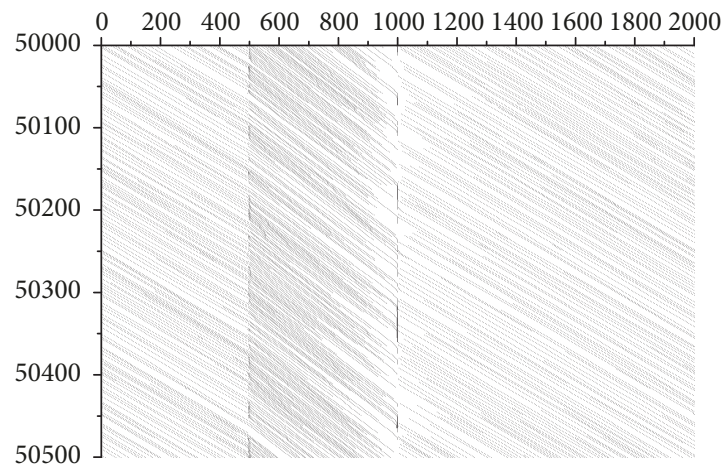

(d) $d_{\mathrm{v} 2 \mathrm{v}}^{1}=500 ; \rho_{\text {max } \mathrm{I}}^{\mathrm{IoV}}=0.14$

FIGURE 9: Spatiotemporal diagrams of different $d_{\mathrm{v} 2 \mathrm{v}}^{1}$ corresponding to $\rho_{\text {max_I }}^{\text {IoV }}$ in IoV-ATCA-AO.

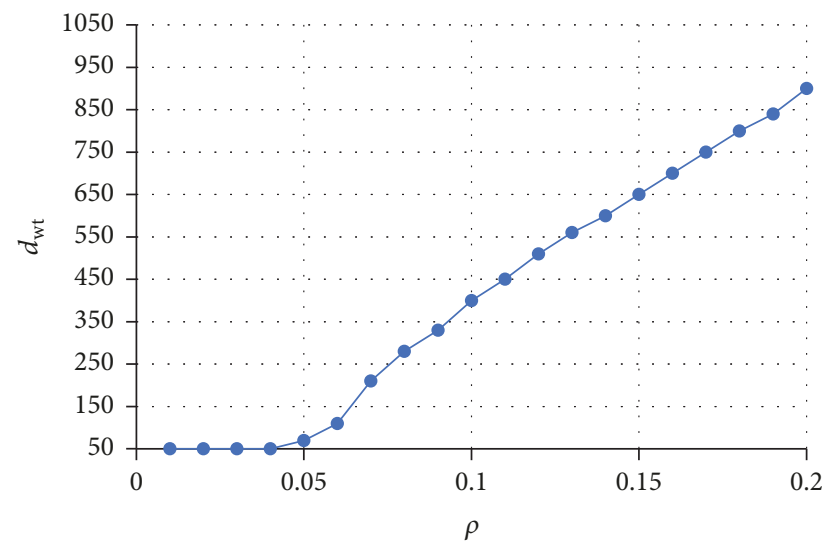

FIGURE 10: Relations between appropriate warning thresholds $d_{\mathrm{wt}}$ and $\rho$.

the two types of accidents are higher. (5) Compared with visual-based avoidance pattern with an abandoned object, the Internet-of-Vehicles-based avoidance pattern with an abandoned object can greatly reduce the rate of both types of accidents. Finally, the effect of Internet-of-Vehicles-based warning threshold with abandoned object on the accident rate is analyzed, and the appropriate warning threshold is also given in different densities to provide reference for early warnings for abandoned objects.

\section{Conflicts of Interest}

The authors declare that there are no conflicts of interest regarding the publication of this paper.

\section{Acknowledgments}

This research work was supported by the National Natural Science Foundation of China (Grant no. 51268051), Natural Science Foundation of Hubei Province (no. 2014CFB287, no. 2013CFA054), and Key Program of Science and Technology of Hubei Provincial Department of Education (no. D20141804).

\section{References}

[1] R. S. Feris and A. Hampapur, "Robust Detection of Abandoned and Removed Objects in Complex Surveillance Videos," IEEE Transactions on Systems Man \& Cybernetics Part C, vol. 41, no. 5, pp. 565-576, 2011.

[2] G. Karagiannis, O. Altintas, E. Ekici et al., "Vehicular networking: a survey and tutorial on requirements, architectures, challenges, standards and solutions," IEEE Communications Surveys \& Tutorials, vol. 13, no. 4, pp. 584-616, 2011.

[3] K. Nagel and M. Schreckenberg, "A cellular automaton model for freeway traffic," Journal de Physique I, vol. 2, no. 12, pp. 22212229, 1992.

[4] M. Takayasu and H. Takayasu, "1/f noise in a traffic model," Fractals, vol. 1, no. 4, pp. 860-866, 1993. 
[5] S. C. Benjamin, N. F. Johnson, and P. M. Hui, "Cellular automata models of traffic flow along a highway containing a junction," Journal of Physics A: Mathematical and General, vol. 29, no. 12, pp. 3119-3127, 1996.

[6] R. Barlovic, L. Santen, A. Schadschneider, and M. Schreckenberg, "Metastable states in cellular automata for traffic flow," The European Physical Journal B: Condensed Matter and Complex Systems, vol. 5, no. 3, pp. 793-800, 1998.

[7] Y. Xue, L.-Y. Dong, and S.-Q. Dai, "An improved onedimensional cellular automaton model of traffic flow and the effect of deceleration probability," Wuli Xuebao/Acta Physica Sinica, vol. 50, no. 3, p. 449, 2001.

[8] X. B. Li, Q. S. Wu, and R. Jiang, "Cellular automaton model considering the velocity effect of a car on the successive car," Physical Review E: Statistical, Nonlinear, and Soft Matter Physics, vol. 64, no. 6, Article ID 066128, 2001.

[9] B. S. Kerner, S. L. Klenov, and D. E. Wolf, "Cellular automata approach to three-phase traffic theory," Journal of Physics A: Mathematical and General, vol. 35, no. 47, pp. 9971-10013, 2002.

[10] R. Jiang and Q.-S. Wu, "Cellular automata models for synchronized traffic flow," Journal of Physics A: Mathematical and General, vol. 36, no. 2, pp. 381-390, 2003.

[11] J.-F. Tian, B. Jia, X.-G. Li, R. Jiang, X.-M. Zhao, and Z.-Y. Gao, "Synchronized traffic flow simulating with cellular automata model," Physica A: Statistical Mechanics and its Applications, vol. 388, no. 23, pp. 4827-4837, 2009.

[12] Z.-T. Xiang, Y.-J. Li, Y.-F. Chen, and L. Xiong, "Simulating synchronized traffic flow and wide moving jam based on the brake light rule," Physica A: Statistical Mechanics and its Applications, vol. 392, no. 21, pp. 5399-5413, 2013.

[13] D. Chowdhury, D. E. Wolf, and M. Schreckenberg, "Particle hopping models for two-lane traffic with two kinds of vehicles: effects of lane-changing rules," Physica A: Statistical Mechanics and its Applications, vol. 235, no. 3-4, pp. 417-439, 1997.

[14] W. Knospe, L. Santen, A. Schadschneider, and M. Schreckenberg, "A realistic two-lane traffic model for highway traffic," Journal of Physics A: Mathematical and General, vol. 35, no. 15, pp. 3369-3388, 2002.

[15] B. Jia, R. Jiang, Q.-S. Wu, and M.-B. Hu, "Honk effect in the two-lane cellular automaton model for traffic flow," Physica A: Statistical Mechanics and its Applications, vol. 348, pp. 544-552, 2005.

[16] X.-G. Li, B. Jia, Z.-Y. Gao, and R. Jiang, "A realistic twolane cellular automata traffic model considering aggressive lane-changing behavior of fast vehicle," Physica A: Statistical Mechanics and its Applications, vol. 367, pp. 479-486, 2006.

[17] B. S. Kerner, S. L. Klenov, and M. Schreckenberg, "Simple cellular automaton model for traffic breakdown, highway capacity, and synchronized flow," Physical Review E: Statistical, Nonlinear, and Soft Matter Physics, vol. 84, no. 4, Article ID 046110, 2011.

[18] H. Y. Shang and Y. Peng, "A new cellular automaton model for traffic flow considering realistic turn signal effect," Science China Technological Sciences, vol. 55, no. 6, pp. 1624-1630, 2012.

[19] H. B. Zhu, N. X. Zhang, and W. J. Wu, "A modified twolane traffic model considering drivers' personality," Physica A: Statistical Mechanics and its Applications, vol. 428, pp. 359-367, 2015.

[20] N. Boccara, H. Fuks, and Q. Zeng, "Car accidents and number of stopped cars due to road blockage on a one-lane highway," Journal of Physics A: Mathematical and General, vol. 30, no. 10, pp. 3329-3332, 1997.
[21] X.-Q. Yang and Y.-Q. Ma, "Car accidents in the deterministic and nondeterministic Nagel-Schreckenberg models," Modern Physics Letters B, vol. 16, no. 9, pp. 333-344, 2002.

[22] R. Jiang, X.-L. Wang, and Q.-S. Wu, "Dangerous situations within the framework of the Nagel-Schreckenberg model," Journal of Physics A: Mathematical and General, vol. 36, no. 17, pp. 4763-4769, 2003.

[23] N. Moussa, "Car accidents in cellular automata models for onelane traffic flow," Physical Review E: Statistical, Nonlinear, and Soft Matter Physics, vol. 68, no. 3, 2003. 


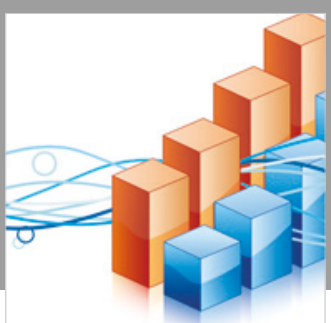

Advances in

Operations Research

\section{-n-m}
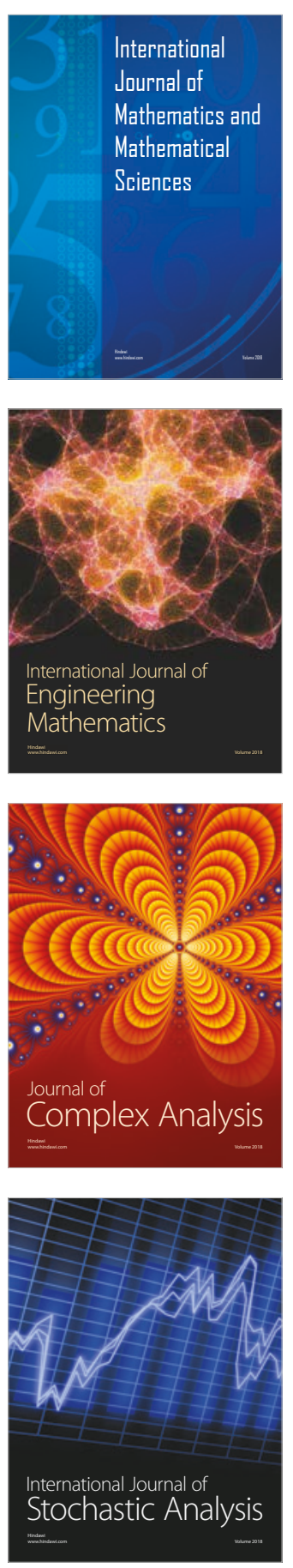
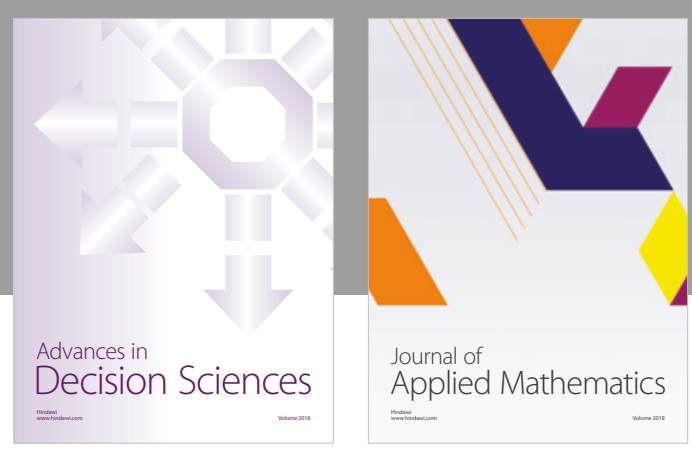

Journal of

Applied Mathematics
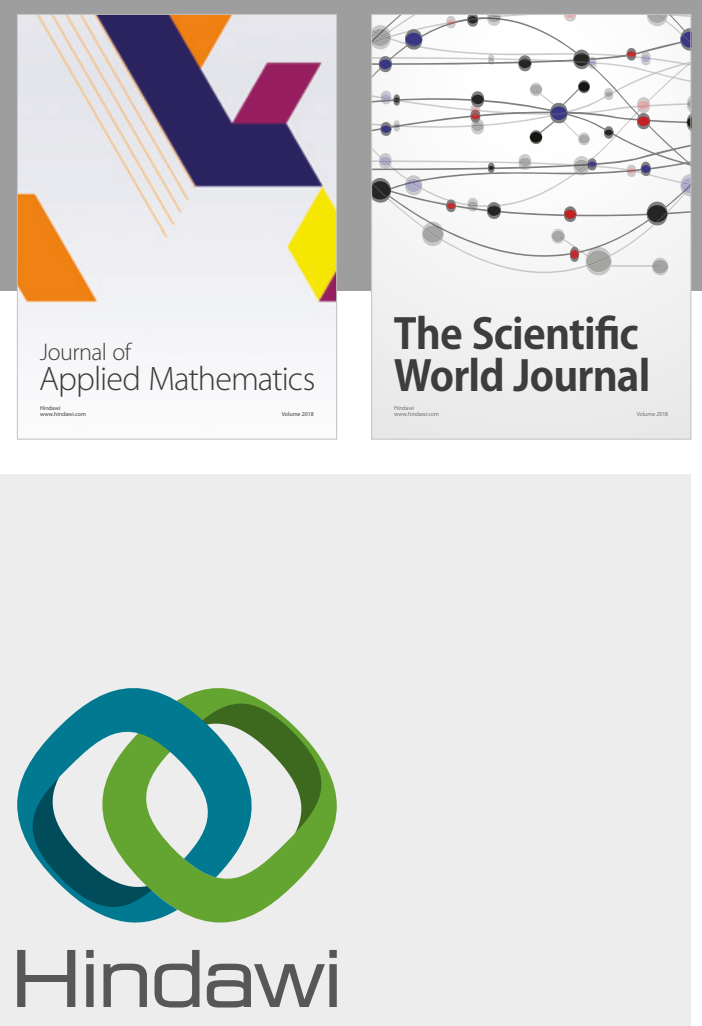

Submit your manuscripts at

www.hindawi.com

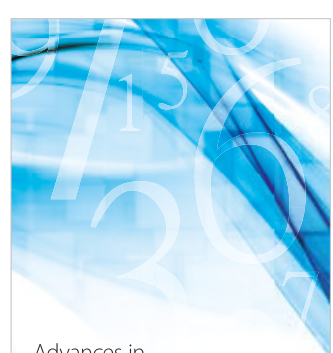

Advances in
Numerical Analysis
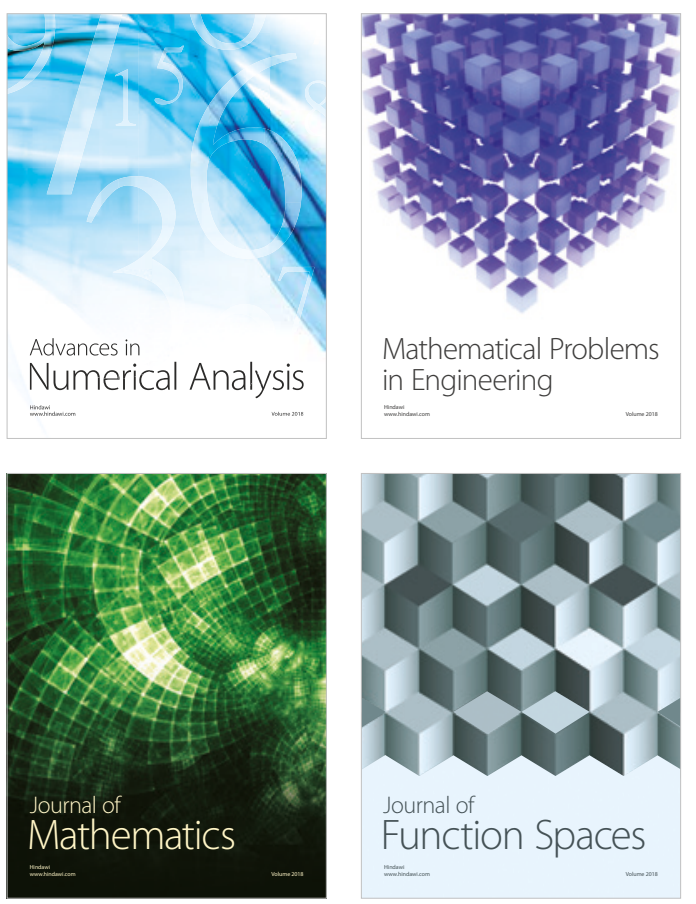

Mathematical Problems in Engineering

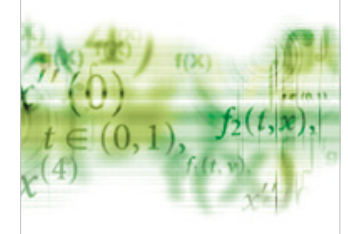

International Journal of

Differential Equations

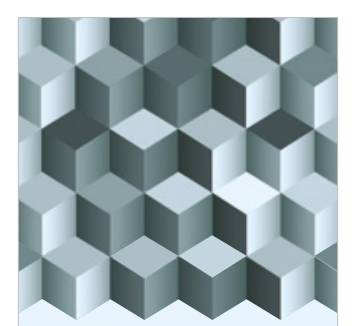

Journal of

Function Spaces

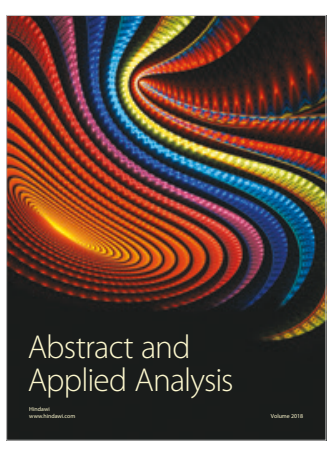

The Scientific

World Journal

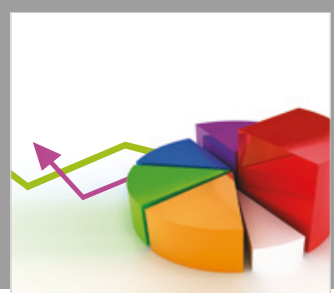

Journal of

Probability and Statistics
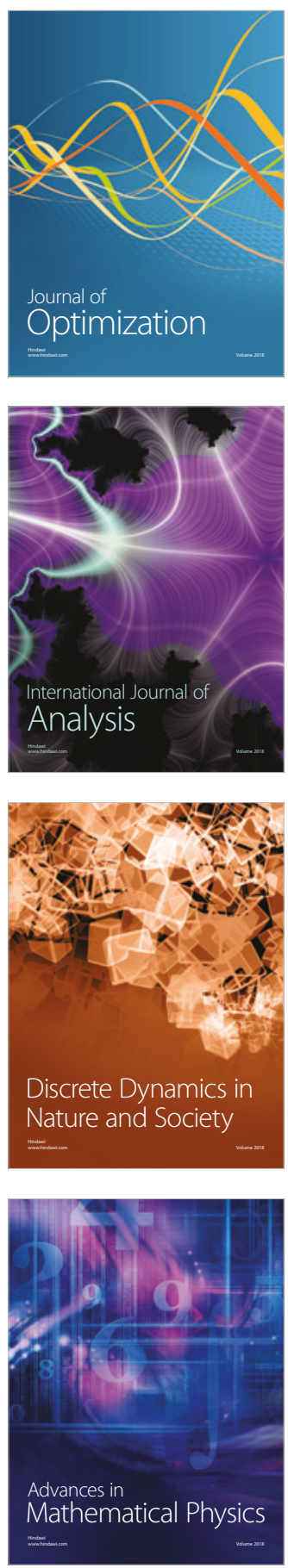